\title{
AN ADAPTIVE UZAWA FEM FOR THE STOKES PROBLEM: CONVERGENCE WITHOUT THE INF-SUP CONDITION*
}

\author{
EBERHARD BÄNSCH ${ }^{\dagger}$, PEDRO MORIN ${ }^{\ddagger}$, AND RICARDO H. NOCHETTO§
}

\begin{abstract}
We introduce and study an adaptive finite element method (FEM) for the Stokes system based on an Uzawa outer iteration to update the pressure and an elliptic adaptive inner iteration for velocity. We show linear convergence in terms of the outer iteration counter for the pairs of spaces consisting of continuous finite elements of degree $k$ for velocity, whereas for pressure the elements can be either discontinuous of degree $k-1$ or continuous of degree $k-1$ and $k$. The popular Taylor-Hood family is the sole example of stable elements included in the theory, which in turn relies on the stability of the continuous problem and thus makes no use of the discrete inf-sup condition. We discuss the realization and complexity of the elliptic adaptive inner solver and provide consistent computational evidence that the resulting meshes are quasi-optimal.
\end{abstract}

Key words. a posteriori error estimators, adaptive mesh refinement, convergence, data oscillation, performance, quasi-optimal meshes

AMS subject classifications. 65N12, 65N15, 65N30, 65N50, 65Y20

PII. S0036142901392134

1. Introduction. Adaptive finite element methods (FEM) have become essential tools in science and engineering for the numerical solution of multiscale phenomena governed by partial differential equations (PDE). We refer to $[1,20]$ for references on adaptivity and restrict the list of papers to those strictly related to our work.

Computational experience strongly suggests that, starting from a coarse mesh, adaptive algorithms converge within any prescribed tolerance in a finite number of steps, but their convergence for general - even linear-problems is largely an open question. This issue has been recently tackled for elliptic problems, in the multidimensional setting, by Morin, Nochetto, and Siebert [15, 16], exploiting an idea of Dörfler [11]. In $[11,15,16]$, the fact that the elliptic operator is positive definite (or coercive) plays a fundamental role.

In this article we devise an adaptive finite element algorithm for the Stokes problem and prove its convergence. The essential difference with elliptic problems is that the Stokes operator is not positive definite but rather leads to a saddle-point problem. The role of coercivity is thus played by the weaker condition of sole invertibility given by the inf-sup condition (1.2) below.

* Received by the editors July 11, 2001; accepted for publication (in revised form) February 11, 2002; published electronically September 27, 2002. This research was partially supported by NSFDAAD grant INT-9910086.

http://www.siam.org/journals/sinum/40-4/39213.html

${ }^{\dagger}$ Weierstrass Institute for Applied Analysis and Stochastics, Mohrenstrasse 39, 10117 Berlin, Germany and Freie Universität Berlin, Arnimallee 2-6, 14195 Berlin, Germany (baensch@ wiasberlin.de).

¥Departamento de Matemática, Facultad de Ingeniería Química, Universidad Nacional del Litoral, 3000 Sante Fe, Argentina and Instituto de Matemática Aplicada del Litoral (IMAL), Güemes 3450, 3000 Santa Fe, Argentina (pmorin@math.unl.edu.ar). The research of this author was partially supported by Programa FOMEC de la Universidad Nacional del Litoral and CONICET of Argentina and NSF grant DMS-9971450. This work was partly developed while this author was visiting the University of Maryland.

$\S$ Department of Mathematics and Institute for Physical Science and Technology, University of Maryland, College Park, MD 20742 (rhn@math.umd.edu). The research of this author was partially supported by NSF grant DMS-9971450. 
To be more specific, let $\Omega$ be a polygonal (polyhedral) domain in $\mathbb{R}^{d}$ for $d \geq 2$, and let $\mathbb{V}=\left(\stackrel{H}{H}^{1}(\Omega)\right)^{d}$ be the usual Sobolev space of vector-valued square integrable functions, having also square integrable first derivatives whose trace vanishes on $\partial \Omega$. Let $\mathbb{P}:=\check{L}^{2}(\Omega)$ be the space of square integrable functions with mean value zero. Then, the weak form of the Stokes problem in its primitive variables reads as follows: Find a pair $(\mathbf{u}, p) \in \mathbb{V} \times \mathbb{P}$ such that for all $(\mathbf{v}, q) \in \mathbb{V} \times \mathbb{P}$,

$$
\int_{\Omega} \nabla \mathbf{u}: \nabla \mathbf{v}-\int_{\Omega} p \operatorname{div} \mathbf{v}=\int_{\Omega} \mathbf{f} \cdot \mathbf{v} \text { and } \int_{\Omega} q \operatorname{div} \mathbf{u}=0,
$$

where throughout this paper we assume $\mathbf{f} \in\left(L^{2}(\Omega)\right)^{d}$. To avoid confusion, vectorvalued functions will always be denoted with boldface characters.

The existence and uniqueness of solutions to (1.1) are equivalent to the so-called inf-sup condition,

$$
\inf _{q \in \mathbb{P}} \sup _{\mathbf{v} \in \mathbb{V}} \frac{\int_{\Omega} q \operatorname{div} \mathbf{v}}{\|q\|\|\nabla \mathbf{v}\|}>0
$$

which holds for the pair $(\mathbb{V}, \mathbb{P})$ as defined above [3]. Hereafter, $\|\cdot\|=\|\cdot\|_{\Omega}$, and for any domain $G,\|w\|_{G}=\left(\int_{G}|w|^{2}\right)^{1 / 2}$ denotes the usual $L^{2}(G)$-norm for scalar- as well as vector- and matrix-valued functions on $G$.

The classical approach to solving the Stokes equations with finite elements is the following. Let $\mathcal{T}_{h}$ be a triangulation of $\Omega$, and let $\mathbb{V}_{h} \subset \mathbb{V}, \mathbb{P}_{h} \subset \mathbb{P}$ be finite element spaces defined on $\mathcal{T}_{h}$. Find a pair $\left(\mathbf{U}_{h}, P_{h}\right) \in \mathbb{V}_{h} \times \mathbb{P}_{h}$ such that

$$
\begin{gathered}
\int_{\Omega} \nabla \mathbf{U}_{h}: \nabla \mathbf{V}_{h}-\int_{\Omega} P_{h} \operatorname{div} \mathbf{V}_{h}=\int_{\Omega} \mathbf{f} \cdot \mathbf{V}_{h} \quad \forall \mathbf{V}_{h} \in \mathbb{V}_{h}, \\
\int_{\Omega} Q_{h} \operatorname{div} \mathbf{U}_{h}=0 \quad \forall Q_{h} \in \mathbb{P}_{h} ;
\end{gathered}
$$

discrete functions will always be written in capitals. Again, this discrete problem admits a unique solution if and only if the discrete inf-sup condition

$$
\inf _{Q_{h} \in \mathbb{P}_{h} \mathbf{V}_{h} \in \mathbb{V}_{h}} \frac{\int_{\Omega} Q_{h} \operatorname{div} \mathbf{V}_{h}}{\left\|Q_{h}\right\|\left\|\nabla \mathbf{V}_{h}\right\|} \geq \kappa>0
$$

holds. Moreover, the following optimal a priori bound holds:

$$
\left\|\nabla\left(\mathbf{u}-\mathbf{U}_{h}\right)\right\|+\left\|p-P_{h}\right\| \leq C_{\kappa}\left(\inf _{\mathbf{V}_{h} \in \mathbb{V}_{h}}\left\|\nabla\left(\mathbf{u}-\mathbf{V}_{h}\right)\right\|+\inf _{Q_{h} \in \mathbb{P}_{h}}\left\|p-Q_{h}\right\|\right),
$$

where $C_{\kappa}$ is a positive constant depending only on $\kappa$ [3]. When a pair of finite element spaces $\left(\mathbb{V}_{h}, \mathbb{P}_{h}\right)$ satisfies (1.5), with $\kappa$ independent of $h$, the method is called stable.

In this article, exploiting an idea introduced in [8] in the context of wavelet approximations to the Stokes problem, we propose and analyze an adaptive FEM for the solution of the Stokes problem. This algorithm consists of an inexact Uzawa iteration at an infinite-dimensional level, and the inner solve is based upon a convergent adaptive FEM for elliptic problems. Amazingly, the convergence of our adaptive Uzawa algorithm (AUA) does not need the discrete inf-sup condition (1.5) but rather the continuous inf-sup condition (1.2). This allows for unstable pairs $\left(\mathbb{V}_{h}, \mathbb{P}_{h}\right)$.

In section 2 we will precisely state the algorithm and prove its convergence for the pairs of spaces consisting of continuous finite elements of degree $k$ for velocity, 
whereas for pressure the elements can be either discontinuous of degree $k-1$ or continuous of degree $k-1$ and $k$. These elements are all unstable, except for the Taylor-Hood elements, which consist of continuous elements of degree $k$ for velocity and degree $k-1$ for pressure. We stress that adaptivity is an inherently nonlinear process, which appears to detect and exploit the stability of the underlying PDE, namely (1.2), regardless of the finite element spaces. This is perhaps the most salient consequence of our work, which reproduces in the finite element setting the crucial observation made in $[7,8]$ for wavelets.

This may seem to contradict the celebrated theory of mixed methods [3]. However, it is important to realize that the $j$ th iterate $\left(\mathbf{U}_{j}, P_{j}\right)$ of our algorithm is not necessarily a solution of the discrete Stokes problem (1.3)-(1.4); it is just an approximate solution. Therefore our notion of convergence is fundamentally different from the customary one arising from a priori error analysis in which (1.5) plays a central role and asymptotics is understood in the sense that the meshsize $h_{j}$ of partition $\mathcal{T}_{j}$ satisfies $h_{j} \rightarrow 0$ : we think of $j \rightarrow \infty$ rather than $h_{j} \rightarrow 0$. Depending on the flatness of $\mathbf{u}$ and $p$, our algorithm may yield convergence even for $h_{j}$ not tending to zero globally.

Although our theory covers only the class of elements mentioned above and described more specifically in (2.5) and (2.6), extensive computations show convergence for other combinations of elements. Moreover, the computational rate of convergence in terms of degrees of freedom is always optimal. This will be discussed in detail in section 3 .

The rest of the article is organized as follows. In section 2 we introduce the AUA and prove its convergence. In section 3 we present numerical evidence showing that the meshes obtained through the AUA are quasi-optimal for any pair of finite element spaces. In section 4 we discuss a posteriori error estimates specially designed for the inexact Uzawa iteration, which are used to stop the outer iterations. Finally, we investigate the properties and complexity of the elliptic inner solver ELLIPTIC in section 5 .

In what follows, unless specified otherwise, $C$ will represent a positive constant, possibly depending on mesh-regularity, and the refinements will be done using bisection $[2,19]$, thus ensuring mesh-regularity.

2. The AUA. We start this section by describing the exact Uzawa algorithm in infinite dimensions as an iteration to solve (1.1). Given $p_{0} \in \mathbb{P}$, we seek, for $j \geq 1$,

$$
\begin{array}{ll}
\mathbf{u}_{j} \in \mathbb{V}: \quad \int_{\Omega} \nabla \mathbf{u}_{j}: \nabla \mathbf{v}=\int_{\Omega} \mathbf{f} \cdot \mathbf{v}+\int_{\Omega} p_{j-1} \operatorname{div} \mathbf{v} \quad \forall \mathbf{v} \in \mathbb{V}, \\
p_{j} \in \mathbb{P}: \quad \int_{\Omega} p_{j} q=\int_{\Omega} p_{j-1} q-\alpha \int_{\Omega} q \operatorname{div} \mathbf{u}_{j} \quad \forall q \in \mathbb{P} .
\end{array}
$$

Recall that $\mathbb{V}=\left(\stackrel{\circ}{H}^{1}(\Omega)\right)^{d}, \mathbb{V}^{*}=\left(H^{-1}(\Omega)\right)^{d}$, and $\mathbb{P}=\stackrel{\circ}{L}^{2}(\Omega)$ and let us denote with $\langle\cdot, \cdot\rangle$ the pairing between $\mathbb{V}$ and $\mathbb{V}^{*}$ as well as the inner product in $\mathbb{P}$. Let us define the operators $-\Delta, \nabla$, and div as follows:

$$
\begin{aligned}
-\Delta: \mathbb{V} \rightarrow \mathbb{V}^{*} \quad\langle-\Delta \mathbf{v}, \mathbf{w}\rangle:=\int_{\Omega} \nabla \mathbf{v}: \nabla \mathbf{w} \quad \forall \mathbf{w} \in \mathbb{V}, \\
\nabla: \mathbb{P} \rightarrow \mathbb{V}^{*} \quad\langle\nabla q, \mathbf{w}\rangle:=-\int_{\Omega} q \operatorname{div} \mathbf{w} \quad \forall \mathbf{w} \in \mathbb{V}, \\
\operatorname{div}: \mathbb{V} \rightarrow \mathbb{P}=\mathbb{P}^{*} \quad\langle\operatorname{div} \mathbf{v}, q\rangle:=\int_{\Omega} q \operatorname{div} \mathbf{v} \quad \forall q \in \mathbb{P} .
\end{aligned}
$$


The Schur complement operator $\mathcal{S}: \mathbb{P} \rightarrow \mathbb{P}$ is defined by

$$
\mathcal{S}:=-\operatorname{div}(-\Delta)^{-1} \nabla
$$

and turns out to be positive definite, self-adjoint, and bounded [6]. Moreover, the Uzawa iteration (2.1) can be written in terms of $\mathcal{S}$ as

$$
p_{j}=(I-\alpha \mathcal{S}) p_{j-1}+\alpha F,
$$

where $F:=-\operatorname{div}(-\Delta)^{-1} \mathbf{f}$. Therefore, if $0<\alpha<2 /\|\mathcal{S}\|_{\mathcal{L}(\mathbb{P}, \mathbb{P})}$, then

$$
\beta:=\|I-\alpha \mathcal{S}\|_{\mathcal{L}(\mathbb{P}, \mathbb{P})}<1
$$

where $\|\cdot\|_{\mathcal{L}(\mathbb{P}, \mathbb{P})}$ denotes the norm in the space of bounded linear operators from the Hilbert space $\mathbb{P}$ into itself. Since $\|\mathcal{S}\|_{\mathcal{L}(\mathbb{P}, \mathbb{P})} \leq 1$ (see [17]), we could take $0<\alpha<2$; we chose $\alpha=1$ in the numerical experiments of section 3 .

From now on, $j \geq 0$ will always denote the Uzawa iteration counter, and $\mathcal{T}_{j}$ will be the $j$ th shape-regular partition of $\Omega$. If $k$ is the polynomial degree for velocity, and $l$ is that for pressure, then we study the pairs of continuous finite element spaces

$$
\mathbb{V}_{j}=\mathcal{P}^{k}\left(\mathcal{T}_{j}\right) \cap \mathbb{V}, \quad \mathbb{P}_{j}=\mathcal{P}^{l}\left(\mathcal{T}_{j}\right) \cap \mathbb{P}, \quad l=k, k-1 \geq 1,
$$

as well as the discontinuous finite element spaces

$$
\mathbb{V}_{j}=\mathcal{P}^{k}\left(\mathcal{T}_{j}\right) \cap \mathbb{V}, \quad \mathbb{P}_{j}=\mathcal{P}_{d}^{k-1}\left(\mathcal{T}_{j}\right) \cap \mathbb{P}, \quad k \geq 1 .
$$

Hereafter, $\mathcal{P}_{d}^{k}\left(\mathcal{T}_{j}\right)$ denotes the space of - scalar-valued as well as vector-valued(possibly discontinuous) functions that, restricted to an element $T$, are polynomials of degree $\leq k$ for all $T \in \mathcal{T}_{j}$, and $\mathcal{P}^{k}\left(\mathcal{T}_{j}\right)$ denotes the subspace of continuous functions of $\mathcal{P}_{d}^{k}\left(\mathcal{T}_{j}\right)$. We observe that $l=k-1$ in (2.5) corresponds to the popular Taylor-Hood family of finite elements. Any other choice in either (2.5) or (2.6) yields an unstable pair of spaces.

Our AUA builds upon a convergent adaptive algorithm for elliptic problems, the procedure ELLIPTIC of section 5, which replaces the first equation in (2.1) by an approximation. To introduce such a procedure, we first consider the following auxiliary elliptic problem: Given $\mathbf{f} \in\left(L^{2}(\Omega)\right)^{d}$ and a pressure function $P_{j-1} \in \mathbb{P}_{j-1}$ for $j \geq 1$, solve

$$
\mathbf{u}_{j} \in \mathbb{V}: \int_{\Omega} \nabla \mathbf{u}_{j}: \nabla \mathbf{v}=\int_{\Omega} \mathbf{f} \cdot \mathbf{v}+\int_{\Omega} P_{j-1} \operatorname{div} \mathbf{v} \quad \forall \mathbf{v} \in \mathbb{V} .
$$

In contrast with (2.1), we observe that $P_{j-1}$ is discrete in (2.7). If $\varepsilon_{j}$ stands for an adjustable error tolerance, then the procedure ELLIPTIC,

$$
\left(\mathcal{T}_{j}, \mathbf{U}_{j}\right) \leftarrow \operatorname{ELLIPTIC}\left(\mathcal{T}_{j-1}, P_{j-1}, \varepsilon_{j}, \mathbf{f}\right),
$$

finds adaptively a refined mesh $\mathcal{T}_{j}$ of $\mathcal{T}_{j-1}$ and solves the discrete elliptic problem

$$
\mathbf{U}_{j} \in \mathbb{V}_{j}: \quad \int_{\Omega} \nabla \mathbf{U}_{j}: \nabla \mathbf{V}=\int_{\Omega} \mathbf{f} \cdot \mathbf{V}+\int_{\Omega} P_{j-1} \operatorname{div} \mathbf{V} \quad \forall \mathbf{V} \in \mathbb{V}_{j},
$$

within the prescribed error bound

$$
\left\|\nabla\left(\mathbf{u}_{j}-\mathbf{U}_{j}\right)\right\| \leq C \varepsilon_{j},
$$


with $C>0$ independent of $j$. We point out that this estimate is not standard in that the right-hand side $\mathbf{f}-\nabla P_{j-1}$ of (2.7) may not be in $L^{2}(\Omega)$ when dealing with discontinuous pressures. This issue is assessed in section 5 .

In addition, let $\Pi_{j}: L^{2}(\Omega) \rightarrow \mathbb{P}_{j}$ denote the orthogonal $L^{2}$-projection into $\mathbb{P}_{j}$. In section 5 , we will show the existence of a constant $C$ independent of $j$ such that the output function $\mathbf{U}_{j}$ of ELLIPTIC satisfies

$$
\left\|\operatorname{div} \mathbf{U}_{j}-\Pi_{j} \operatorname{div} \mathbf{U}_{j}\right\| \leq C \varepsilon_{j} .
$$

The pressure update is performed by the procedure

$$
P_{j} \leftarrow \operatorname{UPDATE}\left(\mathcal{T}_{j}, P_{j-1}, \mathbf{U}_{j}, \alpha\right)
$$

which computes, according to $(2.1)$ with $\mathbb{P}$ replaced by $\mathbb{P}_{j}$,

$$
P_{j} \in \mathbb{P}_{j}: \quad \int_{\Omega} P_{j} Q=\int_{\Omega} P_{j-1} Q-\alpha \int_{\Omega} Q \operatorname{div} \mathbf{U}_{j} \quad \forall Q \in \mathbb{P}_{j},
$$

or equivalently,

$$
P_{j}=P_{j-1}-\alpha \Pi_{j} \operatorname{div} \mathbf{U}_{j} .
$$

We are now in a position to introduce the AUA. This algorithm consists of an inexact inner solve using ELLIPTIC in place of (2.1), followed by an update of pressure given by UPDATE. A similar algorithm was first considered by Dahlke, Hochmuth, and Urban $[8,9]$ in the context of adaptive wavelet methods, which builds upon Elman and Golub [12].

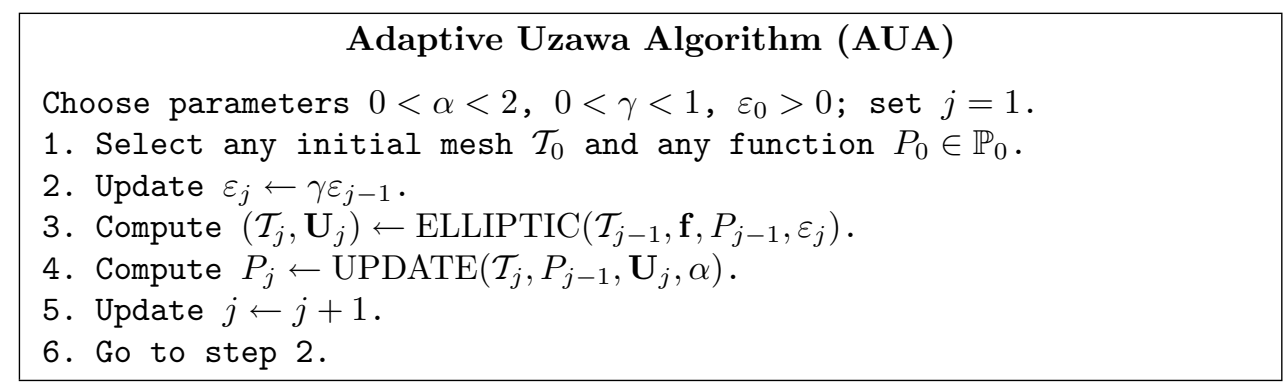

We observe that the AUA makes sense for any pair of spaces $\left(\mathbb{V}_{h}, \mathbb{P}_{h}\right)$, even unstable pairs; this freedom is further investigated in section 3.

Theorem 2.1. Let $\alpha>0$ satisfy (2.4), and let ELLIPTIC fulfill (2.9) and (2.10). Then, there exist positive constants $C_{1}$ and $\delta<1$ such that the iterates $\left(\mathbf{U}_{j}, P_{j}\right)$ produced by the AUA satisfy

$$
\left\|\nabla\left(\mathbf{u}-\mathbf{U}_{j}\right)\right\|+\left\|p-P_{j}\right\| \leq C_{1} \delta^{j} .
$$

Proof. Let us first observe that (2.7) implies $\mathbf{u}_{j}=(-\Delta)^{-1}\left(\mathbf{f}-\nabla P_{j-1}\right)$ for any $j \geq 1$. Hence

$$
\begin{aligned}
P_{j} & =P_{j-1}-\alpha \Pi_{j} \operatorname{div} \mathbf{U}_{j} \\
& =P_{j-1}-\alpha \operatorname{div} \mathbf{u}_{j}+\alpha \operatorname{div}\left(\mathbf{u}_{j}-\mathbf{U}_{j}\right)+\alpha\left(I-\Pi_{j}\right) \operatorname{div} \mathbf{U}_{j} \\
& =(I-\alpha \mathcal{S}) P_{j-1}-\alpha \operatorname{div}(-\Delta)^{-1} \mathbf{f}+\alpha \operatorname{div}\left(\mathbf{u}_{j}-\mathbf{U}_{j}\right)+\alpha\left(I-\Pi_{j}\right) \operatorname{div} \mathbf{U}_{j},
\end{aligned}
$$


where $\mathcal{S}$ stands for the Schur operator (2.2). Analogously, the exact $p$ satisfies

$$
p=(I-\alpha \mathcal{S}) p-\alpha \operatorname{div}(-\Delta)^{-1} \mathbf{f},
$$

which implies

$$
p-P_{j}=(I-\alpha \mathcal{S})\left(p-P_{j-1}\right)-\alpha \operatorname{div}\left(\mathbf{u}_{j}-\mathbf{U}_{j}\right)-\alpha\left(I-\Pi_{j}\right) \operatorname{div} \mathbf{U}_{j} .
$$

Therefore, in view of (2.4), (2.9), and (2.10), together with property $\|\operatorname{div} \mathbf{v}\| \leq\|\nabla \mathbf{v}\|$ [17], we get

$$
\begin{aligned}
\left\|p-P_{j}\right\| & \leq \beta\left\|p-P_{j-1}\right\|+\alpha\left\|\nabla\left(\mathbf{u}_{j}-\mathbf{U}_{j}\right)\right\|+\alpha\left\|\left(I-\Pi_{j}\right) \operatorname{div} \mathbf{U}_{j}\right\| \\
& \leq \beta\left\|p-P_{j-1}\right\|+C \alpha \varepsilon_{j}=\beta\left\|p-P_{j-1}\right\|+C \alpha \varepsilon_{0} \gamma^{j},
\end{aligned}
$$

where $\gamma$ is the reduction factor used in step 2 of the AUA. By induction we obtain

$$
\left\|p-P_{j}\right\| \leq \beta^{j}\left\|p-P_{0}\right\|+C \alpha \varepsilon_{0} \sum_{\ell=0}^{j-1} \beta^{\ell} \gamma^{j-\ell},
$$

and setting $\eta:=\max \{\beta, \gamma\}$, we thus have

$$
\left\|p-P_{j}\right\| \leq\left\|p-P_{0}\right\| \eta^{j}+\alpha \varepsilon_{0} j \eta^{j} \leq C \delta^{j}
$$

for some positive constants $C$ and $\eta<\delta<1$.

To obtain a similar bound for $\left\|\nabla\left(\mathbf{u}-\mathbf{U}_{j}\right)\right\|$, we first observe that

$$
\int_{\Omega} \nabla\left(\mathbf{u}-\mathbf{u}_{j}\right): \nabla \mathbf{v}=\int_{\Omega}\left(p-P_{j-1}\right) \operatorname{div} \mathbf{v} \leq\left\|p-P_{j-1}\right\|\|\nabla \mathbf{v}\| \quad \forall \mathbf{v} \in \mathbb{V},
$$

whence $\left\|\nabla\left(\mathbf{u}-\mathbf{u}_{j}\right)\right\| \leq\left\|p-P_{j-1}\right\|$. Since

$$
\left\|\nabla\left(\mathbf{u}-\mathbf{U}_{j}\right)\right\| \leq\left\|\nabla\left(\mathbf{u}-\mathbf{u}_{j}\right)\right\|+\left\|\nabla\left(\mathbf{u}_{j}-\mathbf{U}_{j}\right)\right\| \leq\left\|p-P_{j-1}\right\|+\varepsilon_{j},
$$

(2.13) yields the desired assertion.

Several comments about the AUA and its convergence properties are now in order.

Remark 2.1. For discontinuous pressure spaces $\mathcal{P}_{d}^{l}\left(\mathcal{T}_{j}\right), l \geq k-1$, the procedure UPDATE of the AUA hinges upon a pressure correction within the subspace $\operatorname{div} \mathbb{V}_{j} \subset$ $\mathcal{P}_{d}^{k-1}\left(\mathcal{T}_{j}\right)$. Consequently, for $l \geq k-1$, the output of the AUA is insensitive to $l$ because the effective pressure space is

$$
\mathbb{P}_{j}=\operatorname{div} \mathbb{V}_{j}
$$

this justifies the restriction $l=k-1$ in (2.6). In contrast, if we enforce continuity of pressure, as in (2.5), then UPDATE works within the subspace $\Pi_{j} \operatorname{div} \mathbb{V}_{j}$ of $\mathbb{P}_{j}=$ $\mathcal{P}^{l}\left(\mathcal{T}_{j}\right)$ for any $l \geq k-1$, and the output of UPDATE does depend on $l$.

Remark 2.2. It is remarkable that the discrete inf-sup condition (1.5) plays no role in our analysis. In fact, the above proof hinges solely on the continuous inf-sup condition (1.2) or, equivalently, on the stability of the infinite-dimensional problem (property $\beta<1$ of $\mathcal{S}$ ). This observation was first made by Dahlke, Hochmuth, and Urban $[8,9]$, and very recently exploited by Dahlke, Dahmen, and Urban [7] and Cohen, Dahmen, and DeVore [5], in the context of wavelet approximations of the Stokes system. 
Remark 2.3. Unstable elements such as (2.6) are known to yield checkerboard patterns in pressure [3]. One may thus wonder whether any adaptive procedure, which extracts discrete regularity via a posteriori error estimation, may be misled by pressure oscillations and thus fail to produce selective local mesh refinement. A possible cure for pressure oscillations within the classical mixed finite element context consists of having a uniformly refined mesh for velocity [3]. In view of (2.8), it turns out that $\left(\mathbf{U}_{j}, P_{j-1}\right)$ is a solution to $(1.3)$ with $P_{j-1}$ defined on a grid $\mathcal{T}_{j-1}$ coarser than $\mathcal{T}_{j}$. This may be regarded as a built-in stabilization, but different from the usual one because $\mathcal{T}_{j}$ is never a global refinement of $\mathcal{T}_{j-1}$ and (1.4) is never fulfilled. This is confirmed by the numerical experiments of section 3, which show optimal meshes for these elements. It thus seems that the nonlinear process associated with adaptivity selects the least amount of refinement necessary to stabilize the method.

Remark 2.4. The procedure ELLIPTIC of the AUA entails an inner loop of the form SOLVE $\rightarrow$ ESTIMATE $\rightarrow$ REFINE for the symmetric and coercive elliptic problem (2.7). To achieve the error reduction of (2.9), two ingredients are necessary. First, we need upper and local lower a posteriori error bounds for (2.8). Second, we need a marking strategy and associated error reduction result (2.9). These issues are discussed in section 5 .

Remark 2.5. Parameters $\alpha, \gamma$, and $\varepsilon_{0}$ control the behavior of the AUA. The convergence of the AUA, but not its rate, is independent of $\gamma$ and $\varepsilon_{0}$ but not of $\alpha$ because it dictates the size of the reduction factor $\beta$ in (2.4). Even though the AUA converges for any choice of $\gamma$ and $\varepsilon_{0}$, provided $0<\gamma<1$ and $\varepsilon_{0}>0$, its performance is greatly influenced by them, especially for unstable elements. In particular, if $\beta<$ $\gamma<1$, then the complexity of ELLIPTIC is independent of $j$, as will be shown in section 5.2.

Remark 2.6. To stop the AUA it is necessary to have a posteriori error estimators especially designed for the pair $\left(\mathbf{U}_{j}, P_{j-1}\right)$, which is not a solution of the discrete Stokes problem over $\mathcal{T}_{j}$. This issue is further investigated in section 4.

3. Experiments and mesh optimality. In this section we focus on the computational performance of the algorithm. We analyze it not only for the elements of Theorem 2.1 but also for cases beyond this. All numerical experiments were carried out using the finite element toolbox ALBERT [18, 19], which provides a flexible programming environment for adaptive finite element computations. Some pictures (Figures 3.2, 3.3, 3.5, 3.7) were produced with the graphics package GRAPE [13].

In order to have an appropriate test bed for the algorithm, we consider two examples in two dimensions and one in three dimensions and run simulations with the AUA for several pairs of elements. They can be divided into three groups, all containing unstable elements: elements of type (2.6), elements of type (2.5) - which include the Taylor-Hood elements - and the continuous unstable pair $\mathcal{P}^{1}-\mathcal{P}^{2}$ which is not covered by our theory. We always use the following parameters and initial guess:

$$
\alpha=1.0, \quad \gamma=0.95, \quad \varepsilon_{0}=2.0, \quad P_{0}=0 .
$$

In order to compare our method with the classical adaptive approach to solving the Stokes equations, we also run experiments with a conventional adaptive strategy of the form SOLVE $\rightarrow$ ESTIMATE $\rightarrow$ REFINE. For these experiments we use the TaylorHood elements $\mathcal{P}^{2}-\mathcal{P}^{1}$ and $\mathcal{P}^{3}-\mathcal{P}^{2}$ and the usual residual-type error estimators. An important difference with the AUA is that in SOLVE we solve the saddle-point problem (1.3), (1.4). 
The comparative results for the three groups of elements as well as for the conventional approach are reported below in Tables 3.1, 3.2, and 3.3. To describe the information they contain, let us assume that we expect a relation of the form

$$
\mathrm{ERR}_{j}:=\left\|\nabla\left(u-U_{j}\right)\right\|+\left\|p-P_{j}\right\| \approx C N_{j}^{-r / d},
$$

where $N_{j}$ denotes the number of degrees of freedom (DOFs) at the step $j$ of the outer loop in the AUA, $r=\min \{k, l+1\}$ is the order of the FEM, and $d$ is the dimension. We then define the experimental orders of convergence $\mathrm{EOC}_{j}$ to be

$$
\mathrm{EOC}_{j}:=-d \frac{\log \left(\mathrm{ERR}_{j} / \mathrm{ERR}_{j-1}\right)}{\log \left(N_{j} / N_{j-1}\right)},
$$

and $\mathrm{EOC}$ to be the asymptotic value of $\mathrm{EOC}_{j}$ for large values of $j$. We also introduce the average error decay (AED) in the energy norm for consecutive outer iterations of the AUA, and the number of DOFs for which the relative energy error

$$
\frac{\left\|\nabla\left(u-U_{j}\right)\right\|+\left\|p-P_{j}\right\|}{\|\nabla u\|+\|p\|}
$$

is less than or equal to prescribed tolerances of $10 \%, 5 \%, 1 \%$, and $0.1 \%$, respectively. To compute the errors, we integrated elementwise using a quadrature rule exact for polynomials up to degree 17 in two dimensions and 7 in three dimensions.

We show pictures of pressure (Figures $3.2,3.3,3.5$ ), the variable most sensitive to instabilities, and corresponding meshes for several elements at $5 \%$ relative accuracy. The velocity never exhibits oscillations, is always well approximated, and is thus not depicted.

We also report curves depicting the relative energy error decay in terms of DOFs and compare them with the optimal slope $-r / d$.

Finally, we draw some conclusions common to all the experiments.

3.1. Example: Smooth solution in two dimensions. Let $\Omega:=(-1,1) \times$ $(-1,1)$ and let the velocity $\mathbf{u}$ and pressure $p$ be given by

$$
\mathbf{u}(x, y):=\left[\begin{array}{c}
2 y \cos \left(x^{2}+y^{2}\right) \\
-2 x \cos \left(x^{2}+y^{2}\right)
\end{array}\right], \quad p=e^{-10\left(x^{2}+y^{2}\right)}-p_{m},
$$

where $p_{m}$ is such that $\int_{\Omega} p=0$ and the forcing $\mathbf{f}$ is computed as $\mathbf{f}=-\Delta u+\nabla p$.

We report the computational results in Table 3.1 and the error decays in Figure 3.1. The behavior of the pressure is illustrated for several pairs of elements in Figures 3.2 and 3.3.

3.2. Example: Singular solution in two dimensions. We consider the Lshaped domain

$$
\Omega:=((-1,1) \times(-1,1)) \backslash([0,1] \times[-1,0])
$$

with reentrant angle $\omega=3 \pi / 2$ at the origin. Let $\alpha \approx 0.544$ be an approximation of the smallest root of the nonlinear equation [10]:

$$
\frac{\sin ^{2}(\alpha \omega)-\alpha^{2} \sin ^{2} \omega}{\alpha^{2}}=0
$$


TABLE 3.1

Example 3.1: EOC, AED per outer iteration, and DOFs to reach tolerances of $10 \%, 5 \%, 1 \%$, $0.1 \%$. The first 7 rows correspond to the AUA and the last 2 to the saddle-point problem (compare them with rows 5 and 6 of the AUA).

\begin{tabular}{|c|c|r|r|r|r|r|}
\hline \multirow{2}{*}{ Spaces } & \multirow{2}{*}{ EOC } & \multirow{2}{*}{ AED } & \multicolumn{4}{|c|}{ DOFs for relative error of } \\
\cline { 4 - 7 } & & & $10 \%$ & $5 \%$ & $1 \%$ & $0.1 \%$ \\
\hline \hline $\mathcal{P}^{1}-\mathcal{P}_{d}^{0}$ & 1.075 & 0.948 & 6570 & 24826 & 448786 & $>10^{6}$ \\
\hline $\mathcal{P}^{2}-\mathcal{P}_{d}^{1}$ & 2.029 & 0.951 & 834 & 1538 & 6930 & 70578 \\
\hline $\mathcal{P}^{3}-\mathcal{P}_{d}^{2}$ & 2.997 & 0.950 & 266 & 1010 & 1754 & 8570 \\
\hline \hline $\mathcal{P}^{1}-\mathcal{P}^{1}$ & 1.044 & 0.948 & 2715 & 9867 & 227991 & $>10^{6}$ \\
\hline $\mathcal{P}^{2}-\mathcal{P}^{1}$ & 1.994 & 0.950 & 295 & 403 & 3403 & 22791 \\
\hline $\mathcal{P}^{3}-\mathcal{P}^{2}$ & 2.878 & 0.952 & 211 & 211 & 947 & 4331 \\
\hline \hline $\mathcal{P}^{1}-\mathcal{P}^{2}$ & 0.905 & 0.950 & 21931 & 109279 & $>10^{6}$ & $>10^{6}$ \\
\hline \hline $\mathcal{P}^{2}-\mathcal{P}^{1}$ & 2.057 & $/$ & 295 & 403 & 3403 & 21351 \\
\hline $\mathcal{P}^{3}-\mathcal{P}^{2}$ & 2.321 & $/$ & 211 & 211 & 947 & 4331 \\
\hline
\end{tabular}
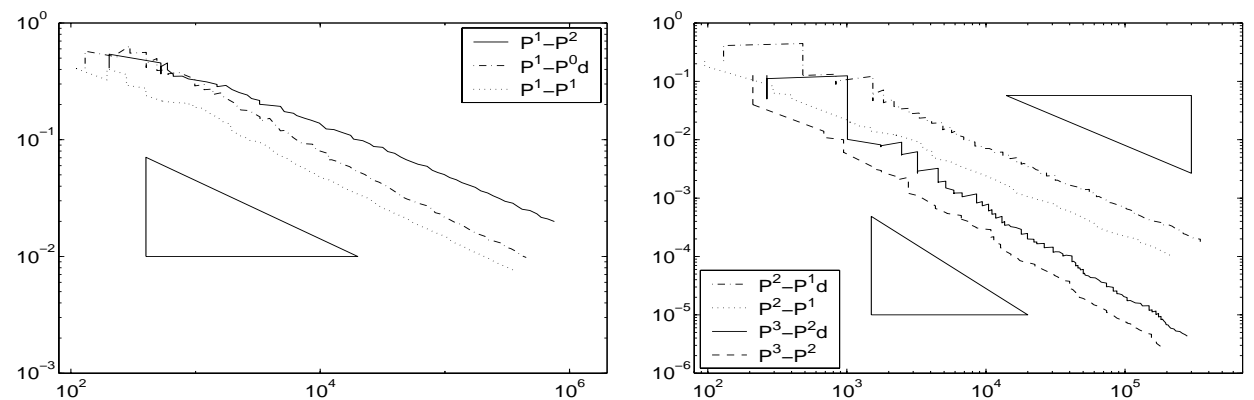

Fig. 3.1. Example 3.1: Relative energy error versus DOFs. Triangles showing optimal decay have slopes $-1 / d=-1 / 2$ (left) and $-2 / d=-1,-3 / d=-3 / 2$ (right), respectively. Quasioptimality of the resulting meshes is thus evident.
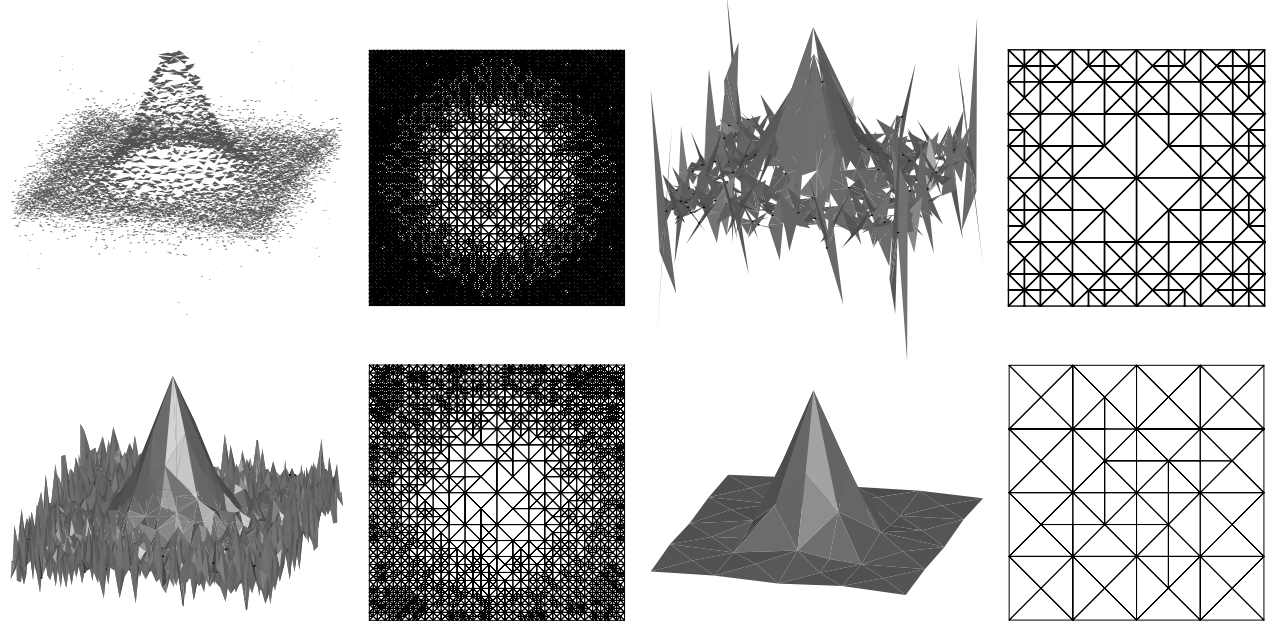

Fig. 3.2. Example 3.1: Pressures and meshes for tolerance of 5\% and finite element pairs (respectively, outer iteration number $/$ DOFs); $\mathcal{P}^{1} \mathcal{P}_{d}^{0}$ (60/24826), $\mathcal{P}^{2}-\mathcal{P}_{d}^{1}(50 / 1538), \mathcal{P}^{1} \mathcal{P}^{1}$ (50/9867), $\mathcal{P}^{2}-\mathcal{P}^{1}(45 / 403)$. The oscillations for unstable pairs do not persist under further selective refinement. 

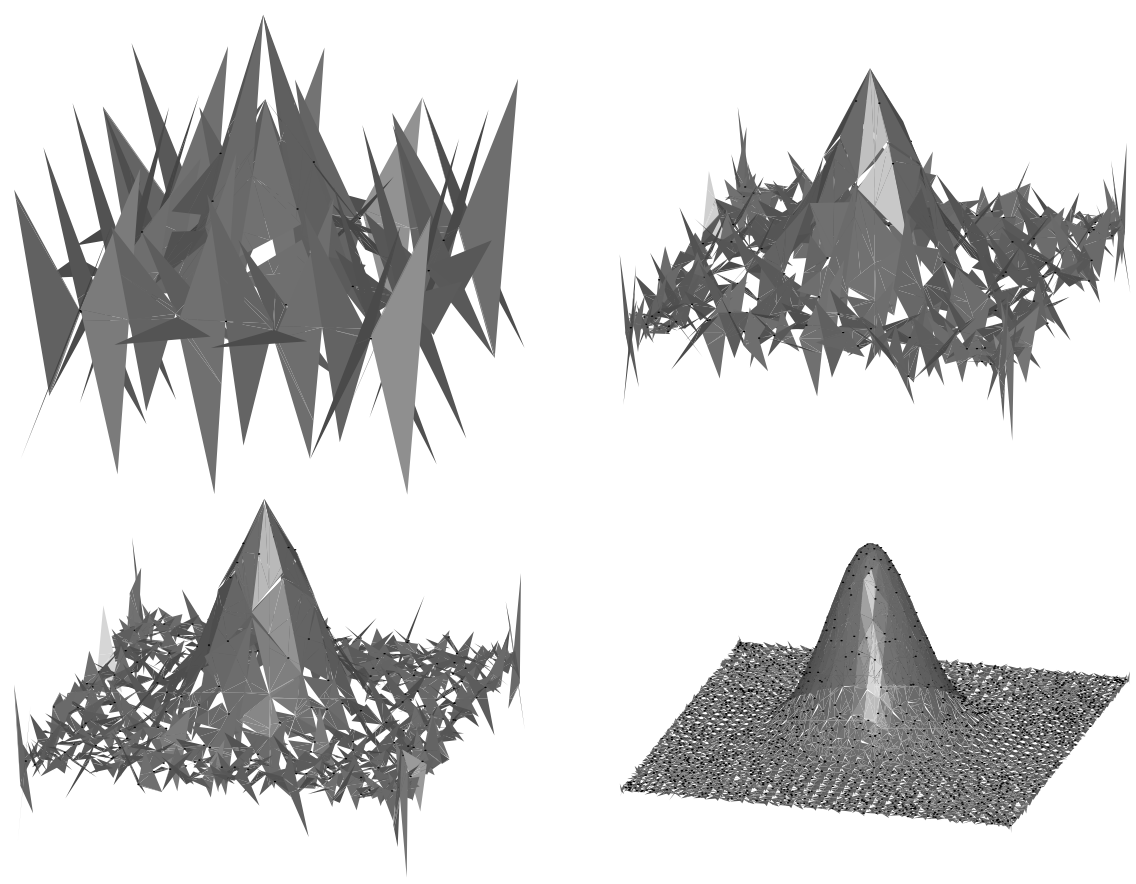

FIG. 3.3. Example 3.1: Sequence of pressures for the unstable pair $\mathcal{P}^{2}-\mathcal{P}_{d}^{1}$ and outer iterations (respectively, DOFs) $j=20($ DOFs $=482), 60$ (2802), 70 (4066), 120 (40986). Oscillations are present in the early stages of adaptivity but are cured later by selective refinement.

The exact velocity $\mathbf{u}$ and pressure $p$ are given in polar coordinates by $[10,21]$

$$
\mathbf{u}(r, \varphi)=r^{\alpha}\left[\begin{array}{l}
\cos (\varphi) \psi^{\prime}(\varphi)+(1+\alpha) \sin (\varphi) \psi(\varphi) \\
\sin (\varphi) \psi^{\prime}(\varphi)-(1+\alpha) \cos (\varphi) \psi(\varphi)
\end{array}\right]=r^{\alpha}\left(\psi^{\prime}(\varphi) \mathbf{e}_{r}-(1+\alpha) \psi(\varphi) \mathbf{e}_{\varphi}\right)
$$

and

$$
p(r, \varphi)=-r^{\alpha-1} \frac{(1+\alpha)^{2} \psi^{\prime}(\varphi)+\psi^{\prime \prime \prime}(\varphi)}{1-\alpha},
$$

where $\psi(\varphi)$ is the function

$$
\begin{aligned}
\psi(\varphi)= & \frac{\sin ((1+\alpha) \varphi) \cos (\alpha \omega)}{1+\alpha}-\cos ((1+\alpha) \varphi) \\
& +\frac{\sin ((\alpha-1) \varphi) \cos (\alpha \omega)}{1-\alpha}+\cos ((\alpha-1) \varphi) .
\end{aligned}
$$

The forcing term is $\mathbf{f}=\mathbf{0}$.

We report the computational results in Table 3.2 and the error decays in Figure 3.4. The behavior of the pressure is illustrated for several pairs of elements in Figure 3.5. In contrast to Example 3.1, the singular nature of $p$ makes selective refinement apparently more effective in this example, which is less prone to oscillations.

3.3. Example: Smooth solution in three dimensions. We consider the cube $\Omega=(-1,1)^{3}$, and the exact velocity $\mathbf{u}$ and pressure $p$,

$$
\mathbf{u}(x, y, z)=\left[\begin{array}{c}
2 y \cos \left(x^{2}+y^{2}\right) \\
-2 x \cos \left(x^{2}+y^{2}\right) \\
0
\end{array}\right], \quad p=\mu e^{-\lambda\left(x^{2}+y^{2}+z^{2}\right)}-p_{m}
$$


TABLE 3.2

Example 3.2: EOC, AED per outer iteration, and DOFs to reach tolerances of $10 \%, 5 \%, 1 \%$, $0.1 \%$. The first 7 rows correspond to the AUA and the last 2 to the saddle-point problem (compare them with rows 5 and 6 of the $A U A)$.

\begin{tabular}{|c|c|r|r|r|r|r|}
\hline \multirow{2}{*}{ Spaces } & \multirow{2}{*}{ EOC } & \multirow{2}{*}{ AED } & \multicolumn{4}{|c|}{ DOFs for relative error of } \\
\cline { 4 - 7 } & & & $10 \%$ & $5 \%$ & $1 \%$ & $0.1 \%$ \\
\hline \hline $\mathcal{P}^{1}-\mathcal{P}_{d}^{0}$ & 1.116 & 0.946 & 3288 & 9680 & 164398 & $>10^{6}$ \\
\hline $\mathcal{P}^{2}-\mathcal{P}_{d}^{1}$ & 1.992 & 0.950 & 1058 & 1940 & 9314 & 85686 \\
\hline $\mathcal{P}^{3}-\mathcal{P}_{d}^{2}$ & 2.984 & 0.950 & 986 & 1598 & 5054 & 20882 \\
\hline \hline $\mathcal{P}^{1}-\mathcal{P}^{1}$ & 1.250 & 0.943 & 1434 & 4971 & 62979 & $>10^{6}$ \\
\hline $\mathcal{P}^{2}-\mathcal{P}^{1}$ & 2.043 & 0.948 & 802 & 1200 & 3913 & 27387 \\
\hline $\mathcal{P}^{3}-\mathcal{P}^{2}$ & 3.182 & 0.948 & 1125 & 1757 & 3153 & 9749 \\
\hline \hline $\mathcal{P}^{1}-\mathcal{P}^{2}$ & 0.907 & 0.948 & 7751 & 41603 & $>10^{6}$ & $>10^{6}$ \\
\hline \hline $\mathcal{P}^{2}-\mathcal{P}^{1}$ & 2.087 & $/$ & 668 & 1012 & 3273 & 26708 \\
\hline $\mathcal{P}^{3}-\mathcal{P}^{2}$ & 3.425 & $/$ & 1125 & 1757 & 3153 & 9985 \\
\hline
\end{tabular}
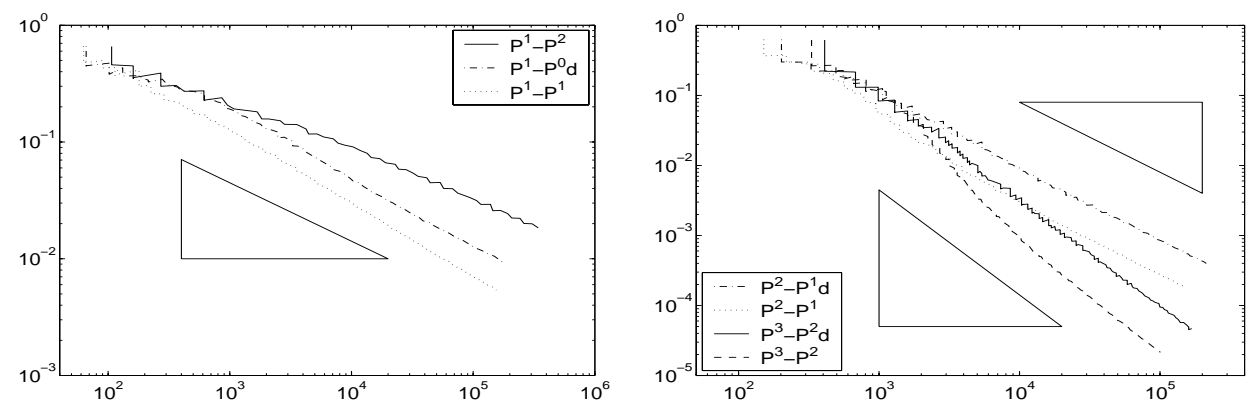

FIG. 3.4. Example 3.2: Relative energy error versus DOFs. Triangles showing optimal decay have slopes $-1 / d=-1 / 2$ (left) and $-2 / d=-1,-3 / d=-3 / 2$ (right), respectively. Quasioptimality of the resulting meshes is thus evident.

where $p_{m}$ is such that $\int_{\Omega} p=0$. The forcing term $\mathbf{f}$ is computed as $\mathbf{f}=-\Delta u+\nabla p$.

We report the computational results in Table 3.3 and the error decays in Figure 3.6, both for $\mu=1, \lambda=10$. Meshes for two finite element pairs are shown in Figure 3.7 for $\mu=10, \lambda=300$.

3.4. Conclusions. We now collect and comment on the consistent information about the AUA extracted from the experiments of sections 3.1-3.3.

- Tables 3.1, 3.2, and 3.3 show that error decay in each outer iteration of the AUA is about 0.95 , regardless of example and pair of elements. This is a consequence of the choice of $\gamma=0.95$ of (3.1) and is further discussed in Remark 5.6. Tables 3.1 and 3.2 also reveal convergence for the unstable pair $\mathcal{P}^{1}-\mathcal{P}^{2}$ in two dimensions, which is not covered by our theory.

- Tables 3.1, 3.2, and 3.3 show that the EOC is optimal for all element pairs and examples and obeys the formula $r=\min \{k, l+1\}$.

- Figures 3.1, 3.4, and 3.6 demonstrate that the relation between error and number of DOFs is optimal for all element pairs and examples: the slopes of the curves match those of the triangles, namely, $-r / d$. The resulting meshes are thus quasi-optimal in all cases. 
TABLE 3.3

Example $3.3(\mu=1, \lambda=10)$ : EOC, AED per outer iteration, and DOFs to reach tolerances of $10 \%, 5 \%, 1 \%, 0.1 \%$. The first 5 rows correspond to the AUA and the last 2 to the saddle-point problem (compare them with rows 4 and 5 of the AUA).

\begin{tabular}{|c|c|r|r|r|r|r|}
\hline \multirow{2}{*}{ Spaces } & \multirow{2}{*}{ EOC } & \multirow{2}{*}{ AED } & \multicolumn{4}{|c|}{ DOFs for relative error of } \\
\cline { 4 - 7 } & & & $10 \%$ & $5 \%$ & $1 \%$ & $0.1 \%$ \\
\hline \hline $\mathcal{P}^{1}-\mathcal{P}_{d}^{0}$ & 1.059 & 0.948 & $>10^{6}$ & $>10^{6}$ & $>10^{6}$ & $>10^{6}$ \\
\hline $\mathcal{P}^{2}-\mathcal{P}_{d}^{1}$ & 1.872 & 0.950 & 23799 & 128903 & $>10^{6}$ & $>10^{6}$ \\
\hline $\mathcal{P}^{3}-\mathcal{P}_{d}^{2}$ & 2.415 & 0.949 & 1509 & 57159 & 320815 & $>10^{6}$ \\
\hline \hline $\mathcal{P}^{2}-\mathcal{P}^{1}$ & 2.149 & 0.951 & 3112 & 10472 & 86316 & $>10^{6}$ \\
\hline $\mathcal{P}^{3}-\mathcal{P}^{2}$ & 3.117 & 0.952 & 1154 & 6736 & 25696 & 136208 \\
\hline \hline $\mathcal{P}^{2}-\mathcal{P}^{1}$ & 2.062 & $/$ & 3112 & 10728 & 71564 & $>10^{6}$ \\
\hline $\mathcal{P}^{3}-\mathcal{P}^{2}$ & 3.239 & $/$ & 1154 & 6736 & 25696 & 136208 \\
\hline
\end{tabular}
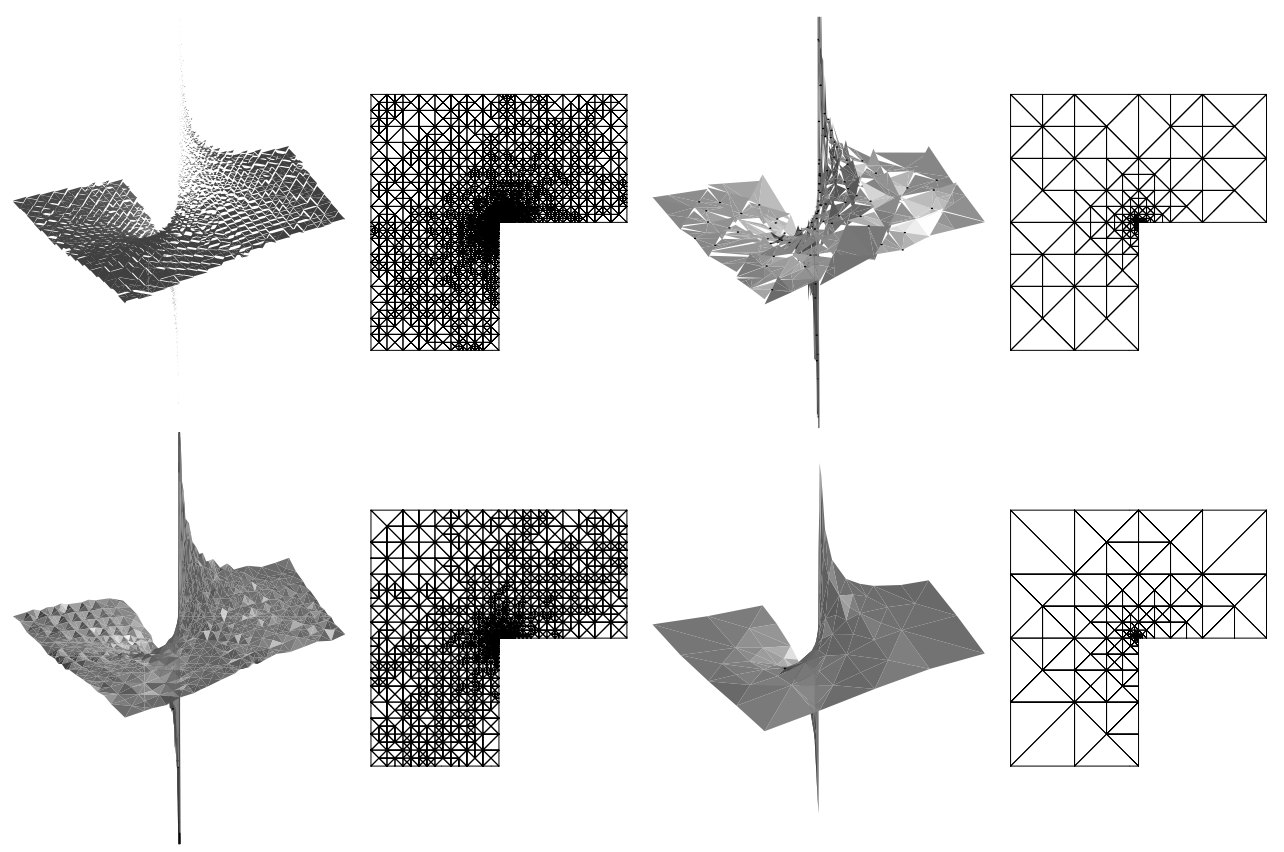

FIG. 3.5. Example 3.2: Pressures and meshes for tolerance of $5 \%$ and finite element pairs (respectively, outer iteration number/DOFs); $\mathcal{P}^{1} \mathcal{P}_{d}^{0}(50 / 9680), \mathcal{P}^{2}-\mathcal{P}_{d}^{1}(35 / 1940), \mathcal{P}^{1} \mathcal{P}^{1}(50 / 4971)$, $\mathcal{P}^{2}-\mathcal{P}^{1}(50 / 1200)$. The oscillations for unstable elements do not persist under further selective refinement.

- Tables 3.1, 3.2, and 3.3 corroborate the fact that higher order elements are superior to lower order elements for piecewise analytic solutions such as those in Examples 3.1-3.3. For a given tolerance, they need many fewer DOFs than lower order elements.

- Tables 3.1, 3.2, and 3.3 display very similar performance between the AUA, with the element pairs of $(2.5)$ and $l=k-1$, and the saddle-point approach with the Taylor-Hood families $\mathcal{P}^{2}-\mathcal{P}^{1}$ and $\mathcal{P}^{3}-\mathcal{P}^{2}$ (last 2 rows of these tables).

- The stable element pairs (2.5) with $l=k-1$ exhibit a slightly better performance than the corresponding unstable pairs (2.6). This is documented in Tables 3.1, 

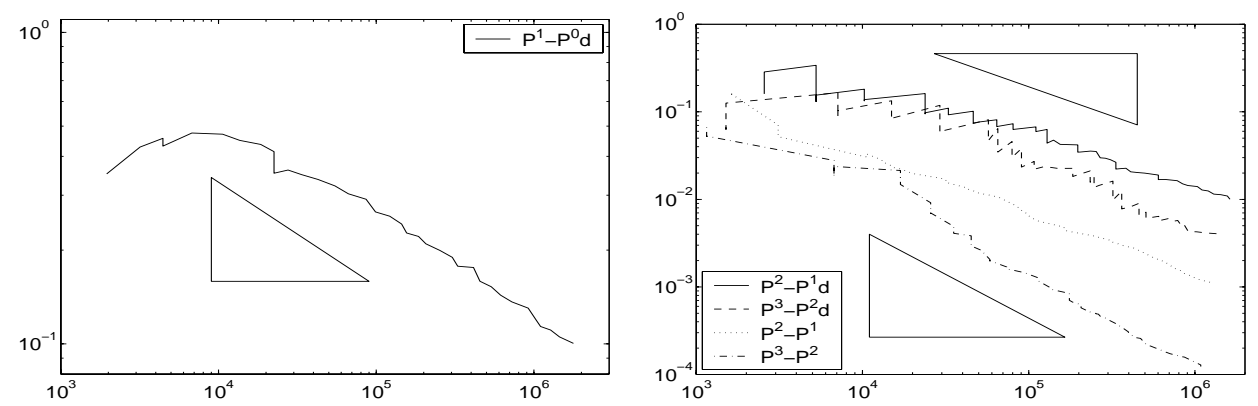

FIG. 3.6. Example $3.3(\mu=1, \lambda=10)$ : Relative energy error versus DOFs. Triangles have slopes $-1 / d=-1 / 3$ (left) and $-2 / d=-2 / 3,-3 / d=-1$ (right), respectively. Quasi-optimality of the resulting meshes is thus evident.
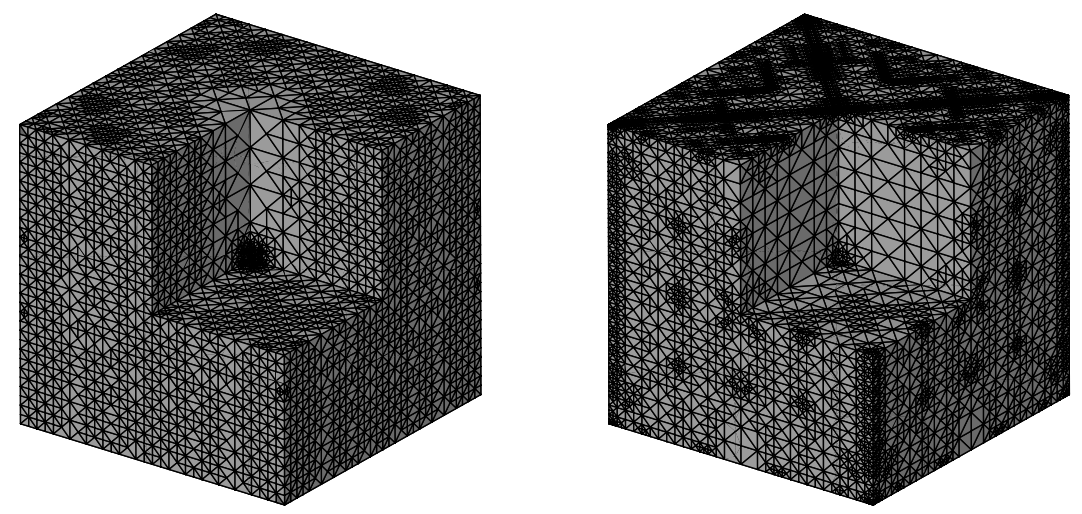

FIG. 3.7. Example $3.3(\mu=10, \lambda=300)$ : Mesh for finite element pair $\mathcal{P}^{2}-\mathcal{P}^{1}$ at outer iteration number $j=105$, DOFs $=1063176$ (left), and mesh for finite element pair $\mathcal{P}^{2}-\mathcal{P}_{d}^{1}$ at $j=70, D O F s$ $=2007799$ (right). The first octant has been removed for visualization purposes.

3.2, and 3.3 in terms of DOFs for a given tolerance, and in Figures 3.2, 3.3, and 3.5 in terms of oscillations.

- It is important to note that oscillations tend to zero in $L^{2}$, thereby giving rise to convergence of pressure in $L^{2}$. However, as suggested by Figures 3.2 and 3.3, this might be a rather weak concept of convergence in practice, which is in contrast to common belief.

4. A posteriori error estimators. In this section we derive a posteriori error estimators for the pair $\left(\mathbf{U}_{j}, P_{j-1}\right)$, which are instrumental to stopping the outer loop in the AUA. We start by defining the bilinear form $\mathcal{L}:(\mathbb{V} \times \mathbb{P}) \times(\mathbb{V} \times \mathbb{P}) \rightarrow \mathbb{R}$,

$$
\mathcal{L}[(\mathbf{v}, q),(\mathbf{w}, r)]:=\int_{\Omega} \nabla \mathbf{v}: \nabla \mathbf{w}-\int_{\Omega} q \operatorname{div} \mathbf{w}+\int_{\Omega} r \operatorname{div} \mathbf{v},
$$

and noting that (1.1) is equivalent to finding a pair $(\mathbf{u}, p) \in \mathbb{V} \times \mathbb{P}$ such that

$$
\mathcal{L}[(\mathbf{u}, p),(\mathbf{v}, q)]=\int_{\Omega} \mathbf{f} \cdot \mathbf{v} \quad \forall(\mathbf{v}, q) \in \mathbb{V} \times \mathbb{P} .
$$


Moreover, the continuous inf-sup condition (1.2) is equivalent to the existence of a constant $\Lambda>0$ such that

$$
\|\nabla \mathbf{v}\|+\|q\| \leq \Lambda \sup _{(\mathbf{w}, r) \in \mathbb{V} \times \mathbb{P}} \frac{\mathcal{L}[(\mathbf{v}, q),(\mathbf{w}, r)]}{\|\nabla \mathbf{w}\|+\|r\|} .
$$

This property alone, or equivalently, the stability of the continuous problem, is responsible for a posteriori error estimates for the Stokes system, even for unstable elements. Therefore, the mere derivation of a posteriori error estimators is no guarantee of convergence of any adaptive algorithm based on them.

In what follows we derive both global upper and local lower a posteriori error bounds for the pair $\left(\mathbf{U}_{j}, P_{j-1}\right)$. This pair is a solution of (2.8), but not of the discrete Stokes problem (1.3)-(1.4) for the following two reasons:

- pressure $P_{j-1}$ is piecewise polynomial in the mesh $\mathcal{T}_{j-1}$, which is coarser than $\mathcal{T}_{j}$

- equation (1.4) is not fulfilled.

Altogether, this makes our error analysis a bit unusual. However, since the same techniques reported in $[1,20]$ apply in our context, we only sketch the proofs for completeness. We first set $\mathbf{e}_{u}:=\mathbf{u}-\mathbf{U}_{j}$ and $e_{p}:=p-P_{j-1}$ and observe that, from (1.1) and (2.8), we have

$$
\mathcal{L}\left[\left(\mathbf{e}_{u}, e_{p}\right),(\mathbf{w}, r)\right]=\sum_{T \in \mathcal{T}_{j}}\left(\int_{T} \mathbf{f} \cdot \mathbf{w}-\left(\nabla \mathbf{U}_{j}-P_{j-1} \mathbf{I}\right): \nabla \mathbf{w}-r \operatorname{div} \mathbf{U}_{j}\right)
$$

for any $\mathbf{w} \in \mathbb{V}, r \in \mathbb{P}$; here $\mathbf{I} \in \mathbb{R}^{d \times d}$ stands for the identity matrix. Since the matrix $\mathbf{T}_{j}:=\nabla \mathbf{U}_{j}-P_{j-1} \mathbf{I}$ plays a crucial role, we introduce the jump residual,

$$
\mathbf{J}_{j}:=\llbracket \mathbf{T}_{j} \cdot \mathbf{n} \rrbracket=\llbracket \nabla \mathbf{U}_{j} \cdot \mathbf{n}-P_{j-1} \mathbf{n} \rrbracket,
$$

which indicates the jump of the vector-valued function $\mathbf{T}_{j} \cdot \mathbf{n}$ across interelement sides $S$. Such a jump is independent of the choice of the normal $\mathbf{n}$ to $S$ and is defined as zero for boundary sides. We also introduce the interior residual,

$$
\mathbf{R}_{j}:=\mathbf{f}+\Delta \mathbf{U}_{j}-\nabla P_{j-1},
$$

which is computed elementwise.

LEMmA 4.1 (upper bound). Let $\left\{\left(\mathbf{U}_{j}, P_{j}\right)\right\}_{j=1}^{\infty}$ be the sequence of solutions produced by the AUA. Then there exists a constant $C^{*}$ depending only on mesh shaperegularity such that the following a posteriori upper bound for the error of the pair $\left(\mathbf{U}_{j}, P_{j-1}\right)$ holds:

$$
\left\|\nabla\left(\mathbf{u}-\mathbf{U}_{j}\right)\right\|+\left\|p-P_{j-1}\right\| \leq C^{*}\left(\sum_{T \in \mathcal{T}_{j}} \zeta_{j}(T)^{2}\right)^{1 / 2}
$$

where the local error indicators $\zeta_{j}(T)$ are given by

$$
\zeta_{j}(T)^{2}=h_{T}^{2}\left\|\mathbf{R}_{j}\right\|_{T}^{2}+h_{T}\left\|\mathbf{J}_{j}\right\|_{\partial T}^{2}+\left\|\operatorname{div} \mathbf{U}_{j}\right\|_{T}^{2} \quad \forall T \in \mathcal{T}_{j},
$$

and the quantity $h_{T}$ represents the diameter of the element $T \in \mathcal{T}_{j}$. 
Proof. Exploiting (2.8), we deduce Galerkin orthogonality $\mathcal{L}\left[\left(\mathbf{e}_{u}, e_{p}\right),(\mathbf{W}, 0)\right]=0$ for all $\mathbf{W} \in \mathbb{V}_{j}$. We then have

$$
\mathcal{L}\left[\left(\mathbf{e}_{u}, e_{p}\right),(\mathbf{w}, r)\right]=\mathcal{L}\left[\left(\mathbf{e}_{u}, e_{p}\right),(\mathbf{w}-\mathbf{W}, r)\right] \quad \forall \mathbf{W} \in \mathbb{V}_{j},
$$

and integrating by parts, with $\mathbf{z}=\mathbf{w}-\mathbf{W}$ we obtain

$$
\mathcal{L}\left[\left(\mathbf{e}_{u}, e_{p}\right),(\mathbf{w}, r)\right]=\sum_{T \in \mathcal{T}_{j}}\left(\int_{T} \mathbf{R}_{j} \mathbf{z}+\frac{1}{2} \int_{\partial T} \mathbf{J}_{j} \mathbf{z}-\int_{T} r \operatorname{div} \mathbf{U}_{j}\right) .
$$

Finally, taking $\mathbf{W} \in \mathbb{V}_{j}$ as the Clément interpolant of $\mathbf{w}$, we arrive at

$$
\|\mathbf{z}\|_{T} \leq C h_{T}\|\nabla \mathbf{w}\|_{\mathcal{N}_{j}(T)}, \quad\|\mathbf{z}\|_{\partial T} \leq C h_{T}^{1 / 2}\|\nabla \mathbf{w}\|_{\mathcal{N}_{j}(T)},
$$

where $\mathcal{N}_{j}(T)$ is the union of all elements of $\mathcal{T}_{j}$ sharing at least a vertex with $T \in \mathcal{T}_{j}$. This, together with (4.1), leads to the assertion.

Remark 4.1. The a posteriori error analysis for the Stokes system is based exclusively on satisfaction of the momentum equation (1.3), or (2.8), but not of the incompressibility equation (1.4). In fact, (1.4) is not valid in either our setting or when stabilizing terms are added [14].

Before stating the local lower error bound, we need to introduce the concept of data oscillation, which accounts for information missing due to the averaging process associated with the FEM. Given a subset of elements $\mathcal{F}$ of $\mathcal{T}_{j}$, we set

$$
\operatorname{osc}(\mathbf{f}, \mathcal{F}):=\left(\sum_{T \in \mathcal{F}} h_{T}^{2}\left\|\mathbf{f}-\mathbf{f}_{T}\right\|_{T}^{2}\right)^{1 / 2},
$$

where $\mathbf{f}_{T}$ is the (local) $L^{2}$-projection of $\mathbf{f}$ into the polynomial space $\mathcal{P}^{k-1}(T)$, and $k$ is the polynomial degree of the velocity space $\mathbb{V}_{j}$. Given an element $T \in \mathcal{T}_{j}$ we designate with $\mathcal{F}_{j}(T)$ either the set of elements of $\mathcal{T}_{j}$ sharing a face with $T$ or their union. This abuse of notation will not lead to confusion.

Lemma 4.2 (lower bound). Let $\left\{\left(\mathbf{U}_{j}, P_{j}\right)\right\}_{j=1}^{\infty}$ be the sequence of solutions produced by the AUA. Then there exists a constant $C_{*}$, depending only on mesh shaperegularity, such that the following local a posteriori lower bound for the error of the $\operatorname{pair}\left(\mathbf{U}_{j}, P_{j-1}\right)$ holds:

$$
\zeta_{j}(T) \leq C_{*}\left(\left\|\nabla\left(\mathbf{u}-\mathbf{U}_{j}\right)\right\|_{\mathcal{F}_{j}(T)}+\left\|p-P_{j-1}\right\|_{\mathcal{F}_{j}(T)}+\operatorname{osc}\left(\mathbf{f}, \mathcal{F}_{j}(T)\right)\right) .
$$

We omit the proof because it is the same as that in $[1,21,20]$. This result shows that the upper bound is sharp (global efficiency), and implies that local efficiency refining where the local indicators $\zeta_{j}(T)$ are large is always necessary to reduce the error. This property seems to be distinctive of finite elements and in fact is not valid for wavelets.

5. ELLIPTIC: Realization and complexity. In this section we first define the procedure ELLIPTIC and prove the key properties (2.9) and (2.10) for the finite element families (2.5) and (2.6). Second, we analyze the complexity of ELLIPTIC in terms of the number of iterations necessary to achieve (2.9). 
5.1. Realization. The study of convergence of adaptive FEM for elliptic problems in the multidimensional setting started with the seminal work by Dörfler [11] and was further developed by Morin, Nochetto, and Siebert in $[15,16]$. In this section we will follow the approach in $[15,16]$ to state the algorithm and prove its convergence for a special class of $H^{-1}$ right-hand sides. This is the class of $L^{2}$ vector-valued functions plus gradients of functions in the pressure space $\mathbb{P}_{h}$ which might have discontinuities across interelement boundaries.

In this section we drop the outer counter $j$ and relabel the input arguments $\mathcal{T}_{j-1}$, $P_{j-1}, \varepsilon_{j}$ of ELLIPTIC as $\mathcal{T}^{0}, P, \varepsilon$ and relabel the output $\mathcal{T}_{j}, \mathbf{U}_{j}$ as $\mathcal{T}$, $\mathbf{U}$. Hence

$$
(\mathcal{T}, \mathbf{U}) \leftarrow \operatorname{ELLIPTIC}\left(\mathcal{T}^{0}, P, \varepsilon, \mathbf{f}\right) .
$$

To avoid confusion we always use superscripts, instead of subscripts, whenever inner iterates of ELLIPTIC are involved. Consequently, for $i \geq 1$ we denote by $\mathcal{T}^{i}$ a refinement of $\mathcal{T}^{i-1}$, by $\mathbb{V}^{i}$ the corresponding finite element space for velocities, and by $\mathbf{U}^{i}$ the solution to the following discrete elliptic problem:

$$
\mathbf{U}^{i} \in \mathbb{V}^{i}: \quad \int_{\Omega} \nabla \mathbf{U}^{i}: \nabla \mathbf{V}=\int_{\Omega} \mathbf{f} \cdot \mathbf{V}+\int_{\Omega} P \operatorname{div} \mathbf{V} \quad \forall \mathbf{V} \in \mathbb{V}^{i} .
$$

This is the discretization of (2.7) or, equivalently, of the elliptic PDE $-\Delta \mathbf{u}=\mathbf{f}-\nabla P$ with $\mathbf{u}=\mathbf{u}_{j}$; since $\mathbf{f}-\nabla P \in \mathbb{V}^{*}$, there exists a unique solution to (2.7). We notice that $P$ does not change with $i$ and that, when ELLIPTIC stops, (5.2) becomes (2.8). According to (4.2) and (4.3), the residuals of (5.2) are

$$
\mathbf{J}:=\llbracket \nabla \mathbf{U}^{i} \cdot \mathbf{n}-P \mathbf{n} \rrbracket, \quad \mathbf{R}:=\mathbf{f}+\Delta \mathbf{U}^{i}-\nabla P .
$$

Lemma 5.1. Let $\mathbf{u}$ be the solution of (2.7). For $i \geq 1$, let $\mathcal{T}^{i}$ be a refinement of $\mathcal{T}^{i-1}$ and let $\mathbf{U}^{i}$ be the solution to (5.2). Let the local error indicators $\eta^{i}(T)$ be

$$
\eta^{i}(T)^{2}:=h_{T}^{2}\|\mathbf{R}\|_{T}^{2}+h_{T}\|\mathbf{J}\|_{\partial T}^{2} \quad \forall T \in \mathcal{T}^{i} .
$$

Then there exist two constants $K^{*}, K_{*}$ depending only on mesh shape-regularity, but otherwise independent of $\mathbf{u}, \mathbf{f}, P$, and $\mathcal{T}^{i}$, such that

$$
\begin{gathered}
\left\|\nabla\left(\mathbf{u}-\mathbf{U}^{i}\right)\right\|^{2} \leq K^{*} \sum_{T \in \mathcal{T}^{i}} \eta^{i}(T)^{2}, \\
\eta^{i}(T)^{2} \leq K_{*}\left(\left\|\nabla\left(\mathbf{u}-\mathbf{U}^{i}\right)\right\|_{\mathcal{F}^{i}(T)}^{2}+\operatorname{osc}\left(\mathbf{f}, \mathcal{F}^{i}(T)\right)^{2}\right) \quad \forall T \in \mathcal{T}^{i} .
\end{gathered}
$$

Proof. We note that the error equation can be written equivalently as

$$
\int_{\Omega} \nabla\left(\mathbf{u}-\mathbf{U}^{i}\right): \nabla \mathbf{v}=\int_{\Omega} \mathbf{f} \cdot \mathbf{v}-\int_{\Omega}\left(\nabla \mathbf{U}^{i}-P \mathbf{I}\right): \nabla \mathbf{v} \quad \forall \mathbf{v} \in \mathbb{V} .
$$

The argument now proceeds as in Lemma 4.1 with $r=0$; see also [20].

Remark 5.1. Regardless of the adaptive algorithm used to reduce the error, the estimate (5.4) allows us to measure the error $\mathbf{u}-\mathbf{U}^{i}$ up to a factor $K^{*}$. Stopping the inner iterations of ELLIPTIC when $\sum_{T \in \mathcal{T}^{i}} \eta^{i}(T)^{2}<\varepsilon^{2}$ thus guarantees (2.9).

To motivate the subsequent discussion about the convergence of adaptive FEM for elliptic problems, we observe that consecutive spaces are nested $\mathbb{V}^{i} \subset \mathbb{V}^{i+1}$, whence $\mathbf{U}^{i}-\mathbf{U}^{i+1} \in \mathbb{V}^{i+1}$. Consequently, using the orthogonal decomposition $\mathbf{u}-\mathbf{U}^{i}=$ $\left(\mathbf{u}-\mathbf{U}^{i+1}\right)+\left(\mathbf{U}^{i+1}-\mathbf{U}^{i}\right)$, the Pythagoras theorem yields

$$
\left\|\nabla\left(\mathbf{u}-\mathbf{U}^{i+1}\right)\right\|^{2}=\left\|\nabla\left(\mathbf{u}-\mathbf{U}^{i}\right)\right\|^{2}-\left\|\nabla\left(\mathbf{U}^{i}-\mathbf{U}^{i+1}\right)\right\|^{2} .
$$


The error reduction is thus exactly $\left\|\nabla\left(\mathbf{U}^{i}-\mathbf{U}^{i+1}\right)\right\|^{2}$. In order to guarantee that the error decreases a fixed proportion of the current error $\left\|\nabla\left(\mathbf{u}-\mathbf{U}^{i}\right)\right\|$, we have to bound $\left\|\nabla\left(\mathbf{U}^{i}-\mathbf{U}^{i+1}\right)\right\|$ from below by $\left\|\nabla\left(\mathbf{u}-\mathbf{U}^{i}\right)\right\|$; this is the key idea from [11]. In view of Lemma 5.1, this reduces to showing a local lower bound for $\left\|\nabla\left(\mathbf{U}^{i}-\mathbf{U}^{i+1}\right)\right\|_{\mathcal{F}^{i}(T)}$ in terms of $\eta^{i}(T)$.

The following lemma states such a lower bound and is crucial for the error reduction property that leads to convergence. Its proof is different from that in [16] due to the presence of the singular term $\nabla P$ in (5.2) and is postponed until the end of this section. We say that an element $T \in \mathcal{T}^{i}$ satisfies the interior node property if

the element $T \in \mathcal{T}^{i}$, as well as each of its faces, contains a node of the finer mesh $\mathcal{T}^{i+1}$ in its interior.

Lemma 5.2. Let $\mathcal{T}^{i+1}$ be a refinement of $\mathcal{T}^{i}$, and let $T \in \mathcal{T}^{i}$ be an element for which every $T^{\prime} \in \mathcal{F}^{i}(T)$ satisfies (5.7). Then, there exists a constant $K_{*}$, depending only on mesh shape-regularity, such that

$$
\eta^{i}(T)^{2} \leq K_{*}\left(\left\|\nabla\left(\mathbf{U}^{i+1}-\mathbf{U}^{i}\right)\right\|_{\mathcal{F}^{i}(T)}^{2}+\operatorname{osc}\left(\mathbf{f}, \mathcal{F}^{i}(T)\right)^{2}\right) .
$$

Remark 5.2. We refer to [15] for a thorough discussion about the requirement (5.7) and its importance for convergence.

We now present the following algorithm, which was first proposed in [15], based on a marking strategy due to Dörfler [11].

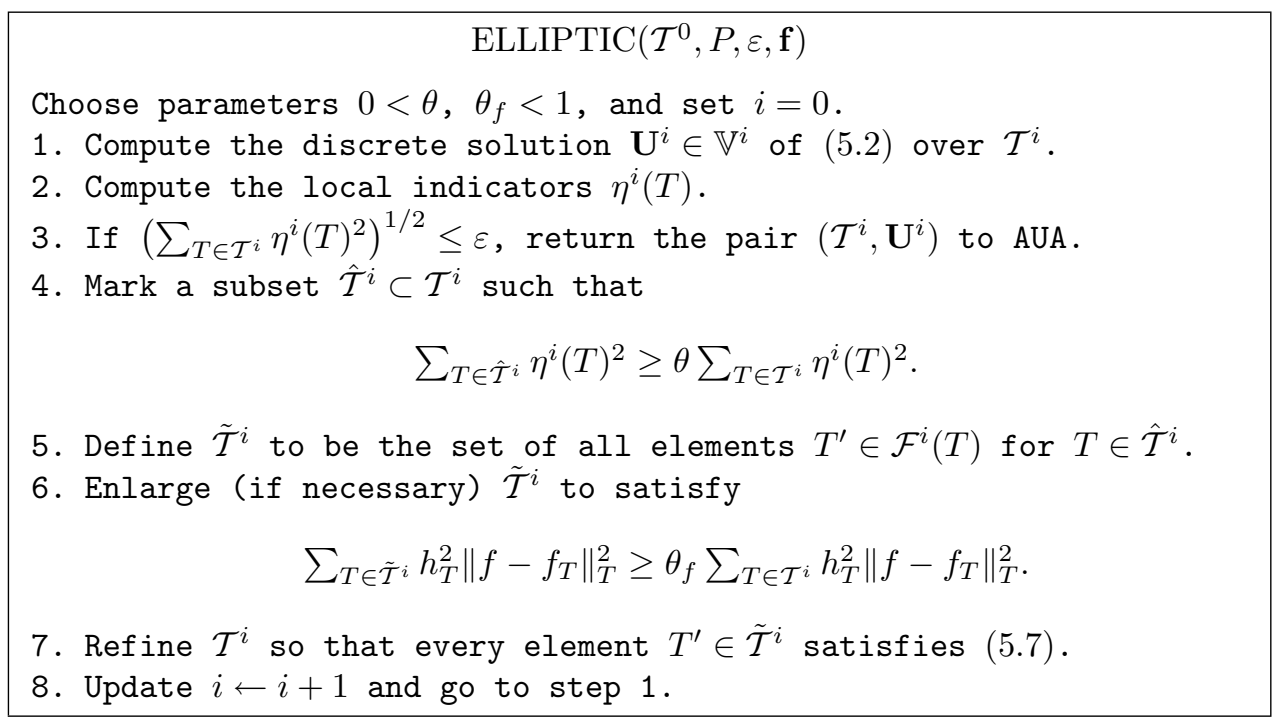

Remark 5.3. Let us note that step 6 implies the existence of a constant $\rho_{f} \in(0,1)$, depending only on $\theta_{f}$ and mesh shape-regularity, such that

$$
\operatorname{osc}\left(f, \mathcal{T}^{i+1}\right) \leq \rho_{f} \operatorname{osc}\left(f, \mathcal{T}^{i}\right) \quad \forall i \geq 0 .
$$

This assertion has been proved in [15] for linear finite elements, but that proof remains valid for any polynomial degree and is thus omitted.

Remark 5.4. Since (5.2) is not the ultimate goal of the AUA, but rather an intermediate problem, we used $\theta=\theta_{f}=0.1$ in the experiments of section 3 for the refinement decisions of ELLIPTIC to be rather conservative. This yields a suitable 
work balance between ELLIPTIC and UPDATE. Moreover, step 6 of ELLIPTIC plays no significant role in practice for smooth $\mathbf{f}[15]$.

For ELLIPTIC we have the following result, which guarantees that (2.9) can be achieved with finite iterations. We show in Proposition 5.6 that this number of iterations is indeed independent of the outer counter $j$.

Proposition 5.3. Let $\mathbf{U}^{i}$ be a sequence of finite element solutions produced by ELLIPTIC. Then, there exist two constants $C_{0}$ and $\rho<1$, depending only on the parameters $\theta, \theta_{f}$ of ELLIPTIC, such that

$$
\left\|\nabla\left(\mathbf{u}-\mathbf{U}^{i}\right)\right\| \leq \rho^{i} \max \left\{\left\|\nabla\left(\mathbf{u}-\mathbf{U}^{0}\right)\right\|, C_{0} \operatorname{osc}\left(f, \mathcal{T}^{0}\right)\right\} .
$$

Proof. By virtue of Lemmas 5.1, 5.2, and step 4 of ELLIPTIC, we have that

$$
\left\|\nabla\left(\mathbf{u}-\mathbf{U}^{i}\right)\right\|^{2} \leq \frac{K^{*}}{\theta} \sum_{T \in \hat{\mathcal{T}}^{i}} \eta^{i}(T)^{2} \leq \frac{1}{\lambda}\left(\left\|\nabla\left(\mathbf{U}^{i}-\mathbf{U}^{i+1}\right)\right\|^{2}+\operatorname{osc}\left(f, \mathcal{T}^{i}\right)^{2}\right),
$$

with $\lambda=\frac{\theta}{(d+2) K^{*} K_{*}}$. Combining this with (5.6) we arrive at

$$
\left\|\nabla\left(\mathbf{u}-\mathbf{U}^{i+1}\right)\right\|^{2} \leq(1-\lambda)\left\|\nabla\left(\mathbf{u}-\mathbf{U}^{i}\right)\right\|^{2}+\operatorname{osc}\left(f, \mathcal{T}^{i}\right)^{2} .
$$

If $\mu>0$ is sufficiently small so that $\rho_{e}^{2}:=1-\lambda+\mu^{2}<1$, as well as

$$
\operatorname{osc}\left(f, \mathcal{T}^{i}\right) \leq \mu\left\|\nabla\left(\mathbf{u}-\mathbf{U}^{i}\right)\right\|,
$$

we then get the error reduction formula

$$
\left\|\nabla\left(\mathbf{u}-\mathbf{U}^{i+1}\right)\right\| \leq \rho_{e}\left\|\nabla\left(\mathbf{u}-\mathbf{U}^{i}\right)\right\| .
$$

To prove (5.9) we set $\rho:=\max \left\{\rho_{e}, \rho_{f}\right\}, C_{0}:=(\mu \rho)^{-1}$, with $\rho_{f}$ as in Remark 5.3, and argue by induction. Since the claim holds trivially for $i=0$, we assume that it holds for $i \geq 0$. Then, we have the two alternatives

$$
\begin{aligned}
& \left\|\nabla\left(\mathbf{u}-\mathbf{U}^{i}\right)\right\|>\rho^{i+1} C_{0} \operatorname{osc}\left(f, \mathcal{T}^{0}\right), \\
& \left\|\nabla\left(\mathbf{u}-\mathbf{U}^{i}\right)\right\| \leq \rho^{i+1} C_{0} \operatorname{osc}\left(f, \mathcal{T}^{0}\right) .
\end{aligned}
$$

In case (5.12), we see from Remark 5.3 that $\operatorname{osc}\left(f, \mathcal{T}^{i}\right) \leq \rho_{f}^{i} \operatorname{osc}\left(f, \mathcal{T}^{0}\right)$, whence

$$
\operatorname{osc}\left(f, \mathcal{T}^{i}\right) \leq \mu \rho^{i+1} \frac{\operatorname{osc}\left(f, \mathcal{T}^{0}\right)}{\rho \mu}=\mu \rho^{i+1} C_{0} \operatorname{osc}\left(f, \mathcal{T}^{0}\right)<\mu\left\|\nabla\left(\mathbf{u}-\mathbf{U}^{i}\right)\right\| .
$$

Consequently, (5.10) holds and, by (5.11) and the induction assumption, we deduce

$$
\left\|\nabla\left(\mathbf{u}-\mathbf{U}^{i+1}\right)\right\| \leq \rho\left\|\nabla\left(\mathbf{u}-\mathbf{U}^{i}\right)\right\| \leq \rho^{i+1} \max \left\{\left\|\nabla\left(\mathbf{u}_{j}-\mathbf{U}^{0}\right)\right\|, C_{0} \operatorname{osc}\left(f, \mathcal{T}^{0}\right)\right\} .
$$

On the other hand, exploiting that $\mathcal{T}^{i+1}$ is a refinement of $\mathcal{T}^{i}$, and thus that the error cannot increase $\left\|\nabla\left(\mathbf{u}-\mathbf{U}^{i+1}\right)\right\| \leq\left\|\nabla\left(\mathbf{u}-\mathbf{U}^{i}\right)\right\|$, we handle (5.13) as follows:

$$
\left\|\nabla\left(\mathbf{u}-\mathbf{U}^{i+1}\right)\right\| \leq \rho^{i+1} C_{0} \operatorname{osc}\left(f, \mathcal{T}_{0}\right) \leq \rho^{i+1} \max \left\{\left\|\nabla\left(\mathbf{u}-\mathbf{U}^{0}\right)\right\|, C_{0} \operatorname{osc}\left(f, \mathcal{T}^{0}\right)\right\} .
$$

The proof is thus complete.

We now verify property (2.10) for the families (2.5) and (2.6). We note that $\operatorname{div} \mathcal{P}^{k}\left(\mathcal{T}_{j}\right) \subset \mathcal{P}_{d}^{k-1}\left(\mathcal{T}_{j}\right)$, whence $\Pi_{j}$ reduces to the identity for (2.6) and thus (2.10) is trivially satisfied. The case (2.5) is more delicate and is the subject of our next result. 
Proposition 5.4. The following interpolation estimate is valid:

$$
\left\|g-\Pi_{j} g\right\| \leq C\left(\sum_{T \in \mathcal{T}_{j}} h_{T}\|\llbracket g \rrbracket\|_{\partial T}^{2}\right)^{1 / 2} \quad \forall g \in \mathcal{P}_{d}^{k-1}\left(\mathcal{T}_{j}\right) .
$$

Proof. We recall that $\Pi_{j} g \in \mathbb{P}_{j}$ is the best $L^{2}$-approximation in $\mathbb{P}_{j}=\mathcal{P}^{l}\left(\mathcal{T}_{j}\right)$ of $g$ and $l \geq k-1$. To prove the assertion we could simply replace $\Pi_{j} g$ by any interpolant of $g$ into $\mathbb{P}_{j}$. We now construct an interpolation operator $I_{j}$ closely related to the Clément operator [4]. Let $\omega_{i}$ be the star of $\mathcal{T}_{j}$ corresponding to the node $x_{i}$, and let $g_{i} \in P^{l}\left(\omega_{i}\right)$ be the $L^{2}$-projection of $g$ into the space of continuous piecewise polynomials $P^{l}\left(\omega_{i}\right)$ :

$$
g_{i} \in P^{l}\left(\omega_{i}\right): \quad \int_{\omega_{i}}\left(g-g_{i}\right) q=0 \quad \forall q \in P^{l}\left(\omega_{i}\right) .
$$

We then set $I_{j} g\left(x_{i}\right):=g_{i}\left(x_{i}\right)$ and recall that to estimate the error $g-I_{j} g$ it suffices to bound $g-g_{i}$ over $\omega_{i}$ for all $i$ [4]. To this end, we first scale $\omega_{i}$ to a reference situation of unit size and then realize that, since $g-g_{i}$ is piecewise polynomial, all its norms are equivalent. In particular, we claim that the seminorm

$$
\left|g-g_{i}\right|_{\omega_{i}}:=\left(\sum_{S \subset \omega_{i}}\|\llbracket g \rrbracket\|_{S}^{2}\right)^{1 / 2}
$$

is a norm. In fact, if $\left|g-g_{i}\right|_{\omega_{i}}=0$ then $g-g_{i}$ is continuous in $\omega_{i}, g-g_{i} \in \mathcal{P}^{l}\left(\omega_{i}\right)$, and thus $g-g_{i}$ is orthogonal to itself, whence $g-g_{i}=0$. A scaling back to $\omega_{i}$ yields the power of meshsize asserted in (5.14) and concludes the proof.

We point out that Proposition 5.4 remains true if $\Pi_{j}$ is an $L^{2}$-projection into any space of continuous piecewise polynomials containing $\mathcal{P}^{k-1}\left(\mathcal{T}_{j}\right)$.

COROLlary 5.5. There exists a constant $C>0$, depending only on mesh shaperegularity and $k$, such that

$$
\left\|\nabla \mathbf{V}-\Pi_{j} \nabla \mathbf{V}\right\| \leq C\left(\sum_{T \in \mathcal{T}_{j}} h_{T}\|\llbracket \nabla \mathbf{V} \cdot \mathbf{n} \rrbracket\|_{\partial T}^{2}\right)^{1 / 2} \quad \forall \mathbf{V} \in \mathcal{P}^{k}\left(\mathcal{T}_{j}\right) .
$$

Proof. We take $g \in \mathcal{P}_{d}^{k-1}\left(\mathcal{T}_{j}\right)$ to be any partial derivative of $\mathbf{V} \in \mathcal{P}^{k}\left(\mathcal{T}_{j}\right)$ and observe that $|\llbracket g \rrbracket| \leq|\llbracket \nabla \mathbf{V} \cdot \mathbf{n} \rrbracket|$ because $V$ being continuous makes the jump $\llbracket \nabla \mathbf{V} \rrbracket$ vanish in any tangential direction. We now apply Proposition 5.4.

To derive (2.10) from Corollary 5.5 in case (2.5), we further note that if $\mathbb{P}_{j-1}$ is a space of continuous finite elements, then the jump residual of (5.3) reduces to the jumps of $\nabla \mathbf{U}_{j}$, which are bounded by $\varepsilon_{j}$ when ELLIPTIC stops.

Proof of Lemma 5.2. We first prove the following estimate for the interior residual $\mathbf{R}$ of (5.3), provided $T \in \mathcal{T}^{i}$ has a node of the finer mesh $\mathcal{T}^{i+1}$ in its interior:

$$
h_{T}^{2}\|\mathbf{R}\|_{T}^{2} \leq C\left(\left\|\nabla\left(\mathbf{U}^{i+1}-\mathbf{U}^{i}\right)\right\|_{T}^{2}+\operatorname{osc}(\mathbf{f}, T)^{2}\right) .
$$

We recall that $\mathbf{f}_{T}$ denotes the orthogonal $L^{2}$-projection of any vector-valued function $\mathbf{f}$ into $\mathcal{P}^{k-1}(T)$. Then, since the degree of the pressure space is $\ell \leq k,\left(\Delta \mathbf{U}^{i}-\nabla P\right)_{T}=$ $\Delta \mathbf{U}^{i}-\nabla P$, whence $\mathbf{R}-\mathbf{R}_{T}=\mathbf{f}-\mathbf{f}_{T}$. 
Now let $\varphi_{T}$ be the canonical continuous piecewise linear basis function of the triangulation $\mathcal{T}^{i+1}$ corresponding to the node inside $T$; thus $\operatorname{supp} \varphi_{T} \subset T$. Since $\mathbf{R}_{T}$ is a polynomial, both $\left\|\mathbf{R}_{T}\right\|_{T}^{2}$ and $\int_{T}\left|R_{T}\right|^{2} \varphi_{T}$ are equivalent up to a constant depending on mesh-regularity. Therefore, integrating by parts and using the fact that $\mathbf{R}_{T} \varphi_{T} \in \mathbb{V}^{i+1}$, we get

$$
\begin{aligned}
\left\|\mathbf{R}_{T}\right\|_{T}^{2} & \leq C \int_{T}\left|\mathbf{R}_{T}\right|^{2} \varphi_{T}=\int_{T} \mathbf{R} \cdot\left(\mathbf{R}_{T} \varphi_{T}\right)+\int_{T}\left(\mathbf{R}_{T}-\mathbf{R}\right) \cdot\left(\mathbf{R}_{T} \varphi_{T}\right) \\
& =\int_{T} \nabla\left(\mathbf{U}^{i+1}-\mathbf{U}^{i}\right): \nabla\left(\mathbf{R}_{T} \varphi_{T}\right)+\int_{T}\left(\mathbf{f}_{T}-\mathbf{f}\right) \cdot\left(\mathbf{R}_{T} \varphi_{T}\right) \\
& \leq C\left(\left\|\nabla\left(\mathbf{U}^{i+1}-\mathbf{U}^{i}\right)\right\|_{T}\left\|\nabla\left(\mathbf{R}_{T} \varphi_{T}\right)\right\|_{T}+\left\|\mathbf{f}_{T}-\mathbf{f}\right\|_{T}\left\|\mathbf{R}_{T} \varphi_{T}\right\|_{T}\right) .
\end{aligned}
$$

Since $\mathbf{R}_{T} \varphi_{T} \in \mathbb{V}^{i+1}$, applying the inverse inequality $\left\|\nabla\left(\mathbf{R}_{T} \varphi_{T}\right)\right\|_{T} \leq C h_{T}^{-1}\left\|\mathbf{R}_{T} \varphi_{T}\right\|_{T}$, together with the triangle inequality $\|\mathbf{R}\|_{T} \leq\left\|\mathbf{R}_{T}\right\|_{T}+\left\|\mathbf{f}-\mathbf{f}_{T}\right\|$, results in (5.15).

We next consider a side $S$ of $\mathcal{T}^{i}$ having a node of $\mathcal{T}^{i+1}$ in its interior and prove the following estimate for the residual $\mathbf{J}$ in (5.3):

$$
h_{T}\|\mathbf{J}\|_{S}^{2} \leq C\left(h_{T}\|\mathbf{R}\|_{\mathcal{F}^{i}(S)}^{2}+\left\|\nabla\left(\mathbf{U}^{i+1}-\mathbf{U}^{i}\right)\right\|_{\mathcal{F}^{i}(S)}^{2}\right) .
$$

Let us first observe that $\mathbf{J}$ is a polynomial of degree at most $k-1$ on $S$. In fact, if $P \in \mathbb{P}=\mathcal{P}_{d}^{k-1}\left(\mathcal{T}_{j}\right)$, then this is obvious (case (2.6)), and if $P \in \mathbb{P}=\mathcal{P}^{l}\left(\mathcal{T}_{j}\right)$, then $P$ does not jump and $\mathbf{J}=\llbracket \nabla \mathbf{U}_{j} \cdot \mathbf{n} \rrbracket$ (case (2.5)). Therefore, $\mathbf{J}$ admits a piecewise polynomial extension to $\mathcal{F}^{i}(S)$ of degree at most $k-1$, which is still denoted by $\mathbf{J}$ (simply scale to the master element and extend $\mathbf{J}$ as a constant in the direction normal to $S)$.

Now let $\varphi_{S}$ be the continuous piecewise linear basis function of the triangulation $\mathcal{T}^{i+1}$ corresponding to the node inside $S$; thus $\operatorname{supp} \varphi_{S} \subset \mathcal{F}^{i}(S)$. Hence $\mathbf{J} \varphi_{S} \in \mathbb{V}^{i+1}$ and $\operatorname{supp}\left(\mathbf{J} \varphi_{S}\right) \subset \mathcal{F}^{i}(S)$. Since $\|\mathbf{J}\|_{S}^{2}$ is equivalent to $\int_{S}|\mathbf{J}|^{2} \varphi_{S}$, integrating by parts and using the fact that $\mathbf{J} \varphi_{S} \in \mathbb{V}^{i+1}$, we obtain

$$
\begin{aligned}
\|\mathbf{J}\|_{S}^{2} & \leq C \int_{S}|\mathbf{J}|^{2} \varphi_{S}=\int_{S} \llbracket \nabla \mathbf{U}^{i} \cdot \mathbf{n}-P \mathbf{n} \rrbracket \cdot \mathbf{J} \varphi_{S} \\
& =-\int_{\mathcal{F}^{i}(S)} \mathbf{R} \cdot \mathbf{J} \varphi_{S}+\int_{\mathcal{F}^{i}(S)} \nabla\left(\mathbf{U}^{i+1}-\mathbf{U}^{i}\right): \nabla\left(\mathbf{J} \varphi_{S}\right) \\
& \leq\|\mathbf{R}\|_{\mathcal{F}^{i}(S)}\left\|\mathbf{J} \varphi_{S}\right\|_{\mathcal{F}^{i}(S)}+\left\|\nabla\left(\mathbf{U}^{i+1}-\mathbf{U}^{i}\right)\right\|_{\mathcal{F}^{i}(S)}\left\|\nabla\left(\mathbf{J} \varphi_{S}\right)\right\|_{\mathcal{F}^{i}(S)} \\
& \leq\left(\|\mathbf{R}\|_{\mathcal{F}^{i}(S)}+\frac{1}{h_{S}}\left\|\nabla\left(\mathbf{U}^{i+1}-\mathbf{U}^{i}\right)\right\|_{\mathcal{F}^{i}(S)}\right)\left\|\mathbf{J} \varphi_{S}\right\|_{\mathcal{F}^{i}(S)} .
\end{aligned}
$$

Multiplying by $h_{S}$ and using the equivalence of $\|\mathbf{J}\|_{S}$ and $\frac{1}{h_{T}}\|\mathbf{J}\|_{\mathcal{F}^{i}(S)}^{2}$, we arrive at the desired estimate (5.16).

To complete the proof, we let $T \in \mathcal{T}^{i}$ satisfy the assumption that all elements $T^{\prime} \in \mathcal{F}^{i}(T)$ possess the interior node property (5.7). We realize that for all those $T^{\prime}$ we can apply (5.15) and next insert the bound for $\left\|\mathbf{R}_{T^{\prime}}\right\|_{T^{\prime}}$ into (5.16).

Remark 5.5. The above proof uncovers the need for the relation $k \geq l$ between the polynomial degrees $k$ for velocity and $l$ for pressure. If this were not true, then $\nabla P$ would differ from $(\nabla P)_{T}$ and we should then account for the oscillation $\nabla P-(\nabla P)_{T}$, which is not given data. Since procedure UPDATE reveals no accuracy gain for $l \geq k$, our assumption $l \leq k$ in (2.5) and (2.6) is not a serious restriction. 
5.2. Complexity. We now turn to the analysis of the complexity of ELLIPTIC. We first observe that the lower bound (5.5), together with the rates of convergence (5.9) and of oscillation reduction (5.8), implies

$$
\begin{aligned}
\sum_{T \in \mathcal{T}^{i}} \eta^{i}(T)^{2} & \leq K_{*}(d+2)\left(\left\|\nabla\left(\mathbf{u}_{j}-\mathbf{U}^{i}\right)\right\|^{2}+\operatorname{osc}\left(\mathbf{f}, \mathcal{T}^{i}\right)^{2}\right) \\
& \leq K_{1} \rho^{2 i}\left\|\nabla\left(\mathbf{u}_{j}-\mathbf{U}^{0}\right)\right\|^{2}+K_{2} \rho^{2 i} \operatorname{osc}\left(\mathbf{f}, \mathcal{T}^{0}\right)^{2}
\end{aligned}
$$

where the constants $K_{1}, K_{2}$ depend only on mesh-regularity and the parameters $\theta$, $\theta_{f}$ of ELLIPTIC. Therefore, the stopping criterion in step 3 of ELLIPTIC can be fulfilled in a finite number of iterations.

A fundamental question that remains open is whether this number can be bounded uniformly with respect to the outer iteration counter $j$. The answer is affirmative and is the subject of the following statement.

Proposition 5.6. Let the tolerance reduction factor $\gamma$ of the AUA satisfy $\gamma>\beta$, where $\beta=\|I-\alpha \mathcal{S}\|_{\mathcal{L}(\mathbb{P}, \mathbb{P})}<1$ is defined in (2.4). Then, the number of iterations in the inner loop of ELLIPTIC is bounded by a constant which depends only on $\mathbf{f}$, the initial pressure guess $P_{0}$, the initial triangulation $\mathcal{T}_{0}$ of the $A U A$, the ratio $\beta / \gamma$, and the parameters $\theta$ and $\theta_{f}$ of ELLIPTIC, but not on the outer index $j$.

Proof. By the preceding comment, the number of iterations of ELLIPTIC is bounded for all outer counters $j$. It is thus sufficient to consider the case $j>1$.

We need to prove the existence of a constant $C$ such that for some $i<C$,

$$
\sum_{T \in \mathcal{T}^{i}} \eta^{i}(T)^{2} \leq \varepsilon^{2}
$$

We recall that the initial mesh $\mathcal{T}^{0}$ of the inner loop is always taken to be the mesh $\mathcal{T}_{j-1}$ of the previous outer loop. Since the term $\Delta \mathbf{U}_{j}-\nabla P_{j-1}$ of the interior residual $\mathbf{R}_{j-1}$ does not oscillate, using definition (4.4), we get

$$
\begin{aligned}
\operatorname{osc}\left(\mathbf{f}, \mathcal{T}^{0}\right) & =\operatorname{osc}\left(\mathbf{R}_{j-1}, \mathcal{T}_{j-1}\right) \leq\left(\sum_{T \in \mathcal{T}_{j-1}} h_{T}^{2}\left\|\mathbf{R}_{j-1}\right\|_{T}^{2}\right)^{1 / 2} \\
& \leq\left(\sum_{T \in \mathcal{T}_{j-1}} h_{T}^{2}\left\|\mathbf{R}_{j-1}\right\|_{T}^{2}+h_{T}\left\|\mathbf{J}_{j-1}\right\|_{\partial T}^{2}\right)^{1 / 2} \\
& =\left(\sum_{T \in \mathcal{T}_{j-1}} \eta_{j-1}(T)^{2}\right)^{1 / 2} \leq \varepsilon_{j-1}=\varepsilon_{0} \gamma^{j-1}
\end{aligned}
$$

where the last inequality is guaranteed by the stopping criterion (step 3) of ELLIPTIC. This accounts for the second term in (5.17).

To estimate $\left\|\nabla\left(\mathbf{u}_{j}-\mathbf{U}^{0}\right)\right\|$ in (5.17), we first split it into three parts:

$$
\left\|\nabla\left(\mathbf{u}_{j}-\mathbf{U}^{0}\right)\right\| \leq\left\|\nabla\left(\mathbf{u}_{j}-\mathbf{u}_{j-1}\right)\right\|+\left\|\nabla\left(\mathbf{u}_{j-1}-\mathbf{U}_{j-1}\right)\right\|+\left\|\nabla\left(\mathbf{U}_{j-1}-\mathbf{U}^{0}\right)\right\| .
$$

By virtue of (2.9), we have

$$
\left\|\nabla\left(\mathbf{u}_{j-1}-\mathbf{U}_{j-1}\right)\right\| \leq C \varepsilon_{j-1}=C \varepsilon_{0} \gamma^{j-1} .
$$



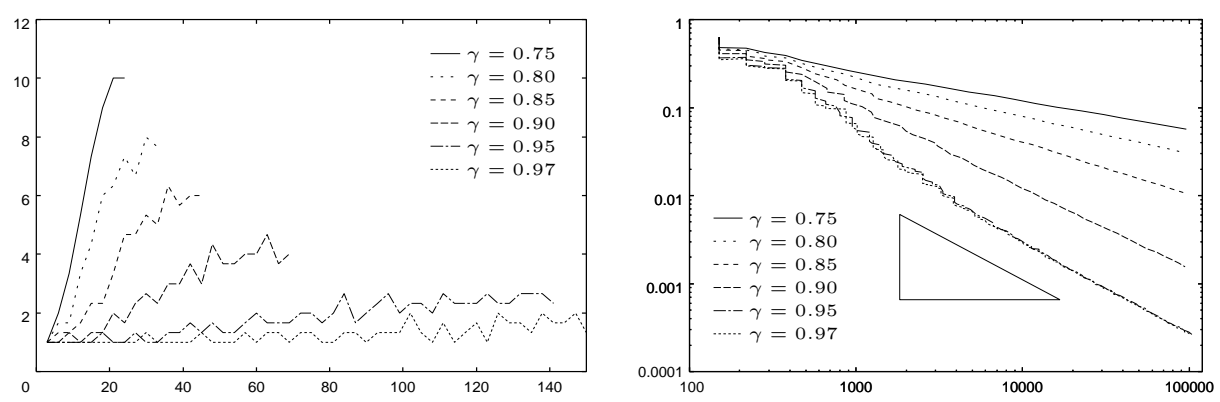

FIG. 5.1. Example 3.2: Number of inner iterations versus outer interations (left) and error decay versus DOFs (right) for different values of $\gamma$. A triangle shows the optimal decay.

Since $\mathbb{V}^{0}=\mathbb{V}_{j-1}$, both $\mathbf{U}^{0}$ and $\mathbf{U}_{j-1}$ belong to $\mathbb{V}_{j-1}$ and, by (2.8), they satisfy

$$
\int_{\Omega} \nabla\left(\mathbf{U}^{0}-\mathbf{U}_{j-1}\right): \nabla \mathbf{V}=\int\left(P_{j-1}-P_{j-2}\right) \operatorname{div} \mathbf{V} \quad \forall \mathbf{V} \in \mathbb{V}_{j-1} .
$$

Hence, taking $\mathbf{V}=\mathbf{U}^{0}-\mathbf{U}_{j-1}$ implies

$$
\left\|\nabla\left(\mathbf{U}_{j-1}-\mathbf{U}^{0}\right)\right\| \leq\left\|P_{j-1}-P_{j-2}\right\| \leq\left\|P_{j-1}-p\right\|+\left\|p-P_{j-2}\right\| .
$$

A similar energy argument, based on the fact that $\mathbf{u}_{j}$ is the solution to (2.7), yields the same estimate for $\left\|\nabla\left(\mathbf{u}_{j}-\mathbf{u}_{j-1}\right)\right\|$. To derive a suitable estimate for $\left\|p-P_{j}\right\|$, we now improve (2.12) as follows:

$$
\left\|p-P_{j}\right\| \leq \beta^{j}\left\|p-P_{0}\right\|+C \alpha \varepsilon_{0} \sum_{\ell=0}^{j-1} \beta^{\ell} \gamma^{j-\ell} \leq \beta^{j}\left\|p-P_{0}\right\|+C \alpha \varepsilon_{0} \gamma^{j} \frac{1-(\beta / \gamma)^{j}}{1-(\beta / \gamma)} .
$$

Inserting the previous estimates back into (5.17), we find a constant $K$, depending on $f$, the initial pressure guess $P_{0}$, the initial triangulation $\mathcal{T}_{0}$ of the AUA, the parameters $\theta, \theta_{f}$ of ELLIPTIC, and the ratio $\beta / \gamma<1$, such that

$$
\left(\sum_{T \in \mathcal{T}^{i}} \eta^{i}(T)^{2}\right)^{1 / 2} \leq K \rho^{i} \gamma^{j} .
$$

Therefore, $K \rho^{i} \gamma^{j} \leq \varepsilon_{j}=\varepsilon_{0} \gamma^{j}$ whenever $\rho^{i} \leq \varepsilon_{0} / K$, and the assertion is proved.

Remark 5.6. It might seem at first sight that $\gamma>\beta$ is an artificial requirement of the proof and thus that the result should still hold for any $\gamma<1$. If $\gamma<\beta$, then the above proof would also give (5.18) with $\beta$ instead of $\gamma$, whence

$$
i \leq C_{1} j+C_{2}
$$

for appropriate constants $C_{1}, C_{2}>0$. This linear growth is corroborated by the simulations leading to Figure 5.1, which depicts the number of inner loops $i$ versus the outer loop counter $j$ for Example 3.2 with the Taylor-Hood element $\mathcal{P}^{2}-\mathcal{P}^{1}$.

Remark 5.7. Since $\beta$ is not known in general, the requirement $\beta<\gamma<1$ may seem restrictive in practice. On the other hand, a value of $\gamma$ too close to 1 results in a large number of outer iterations. We found a practical compromise $\gamma=0.95$ for all simulations of section 3 that leads to a number of inner iterations between 3 and 5 . 
Acknowledgment. The authors would like to thank Kunibert G. Siebert for his assistance with the implementation of discontinuous finite elements within ALBERT and for many valuable suggestions and discussions about this work.

\section{REFERENCES}

[1] M. Ainsworth and J. Oden, A Posteriori Error Estimation in Finite Element Analysis, John Wiley, New York, 2000.

[2] E. Bänsch, Local mesh refinement in 2 and 3 dimensions, Impact Comput. Sci. Engrg., 3 (1991), pp. 181-191.

[3] F. Brezzi And M. Fortin, Mixed and Hybrid Finite Element Methods, Springer-Verlag, Berlin, 1991.

[4] P. ClÉment, Approximation by finite element functions using local regularizations, Rev. Française Automat. Informat. Recherche Opérationalle Sér. Rouge Anal. Numér., 9 (1975), pp. $77-84$.

[5] A. Cohen, W. Dahmen, And R. DeVore, Adaptive Wavelet Methods II-Beyond the Elliptic Case, IGPM Report, Rheinisch-Westfälische Technische Hochschule (RWTH), Aachen, Germany, 2000.

[6] M. Crouzeix, Etude d'une méthode de linéarisation. Résolution numérique des équations de Stokes stationnaires. Application aux équations de Navier-Stokes stationnaires, in Approximation et Méthodes Itératives de Résolution d'Inéquations Variationnelles et de Problèmes Non Linéaires, IRIA Cahier 12, Inst. Recherche Informat. Automat., Rocquencourt, France, 1974, p. 139.

[7] S. Dahlke, W. Dahmen, And K. URBan, Adaptive wavelet methods for saddle point problemsoptimal convergence rates, SIAM J. Numer. Anal., 40 (2002), pp. 1230-1262.

[8] S. Dahlke, R. Hochmuth, and K. Urban, Adaptive wavelet methods for saddle point problems, M2AN Math. Model. Numer. Anal., 34 (2000), pp. 1003-1022.

[9] S. Dahlke, R. Hochmuth, and K. Urban, Convergent adaptive wavelet methods for the Stokes problem, in Multigrid Methods VI, E. Dick, K. Riemslagh, and J. Vierendeels, eds., Springer-Verlag, Berlin, 2000, pp. 66-72.

[10] M. Dauge, Stationary Stokes and Navier-Stokes systems on two- or three-dimensional domains with corners. Part I: Linearized equations, SIAM J. Math. Anal., 20 (1989), pp. 74-97.

[11] W. Dörfler, A convergent adaptive algorithm for Poisson's equation, SIAM J. Numer. Anal., 33 (1996), pp. 1106-1124.

[12] H.C. Elman And G.H. Golub, Inexact and preconditioned Uzawa algorithms for saddle-point problems, SIAM J. Numer. Anal., 31 (1994), pp. 1645-1661.

[13] GRAPE-GRAphics Programming Environment, Manual, Version 5.0 SFB 256, University of Bonn, Bonn, Germany, 1995.

[14] D. KAY AND D. Silvester, A posteriori error estimation for stabilized mixed approximations of the Stokes equations, SIAM J. Sci. Comput., 21 (1999), pp. 1321-1336.

[15] P. Morin, R.H. Nochetto, And K.G. Siebert, Data oscillation and convergence of adaptive FEM, SIAM J. Numer. Anal., 38 (2000), pp. 466-488.

[16] P. Morin, R.H. Nochetto, And K.G. Siebert, Basic Principles for Convergence of Adaptive Higher-Order FEM, manuscript.

[17] J-H. Pyo, The Gauge-Uzawa and Related Projection Finite Element Methods for the Evolution Navier-Stokes Equations, Ph.D. Dissertation, University of Maryland, College Park, MD, 2002.

[18] A. Schmidt And K.G. Siebert, ALBERT-software for scientific computations and applications, Acta Math. Univ. Comenian. (N.S.), 70 (2000), pp. 105-122.

[19] A. Schmidt And K.G. Siebert, Concepts of the finite element toolbox ALBERT, Notes Numer. Fluid Mech., to appear.

[20] R. Verfürth, A Review of A Posteriori Error Estimation and Adaptive Mesh-Refinement Techniques, Wiley, New York, 1996.

[21] R. Verfürth, A posteriori error estimators or the Stokes equations, Numer. Math., 55 (1989), pp. 309-325. 